\title{
Long-Term Observations Of The Pulsars In 47 Tucanae - li. Proper Motions, Accelerations And Jerks
}

\author{
P. C. C. Freire
}
A. Ridolfi
M. Kramer
D. R. Lorimer

Follow this and additional works at: https://researchrepository.wvu.edu/faculty_publications

\section{Digital Commons Citation}

Freire, P. C. C.; Ridolfi, A.; Kramer, M.; and Lorimer, D. R., "Long-Term Observations Of The Pulsars In 47 Tucanae - li. Proper Motions, Accelerations And Jerks" (2017). Faculty \& Staff Scholarship. 989. https://researchrepository.wvu.edu/faculty_publications/989

This Article is brought to you for free and open access by The Research Repository @ WVU. It has been accepted for inclusion in Faculty \& Staff Scholarship by an authorized administrator of The Research Repository @ WVU. For more information, please contact beau.smith@mail.wvu.edu. 


\title{
Long-term observations of the pulsars in 47 Tucanae - II. Proper motions, accelerations and jerks
}

\author{
P. C. C. Freire ${ }^{1 \star}$, A. Ridolfi ${ }^{1}$, M. Kramer ${ }^{1}$, C. $\operatorname{Jordan}^{2}$, R. N. Manchester ${ }^{3}$, P.

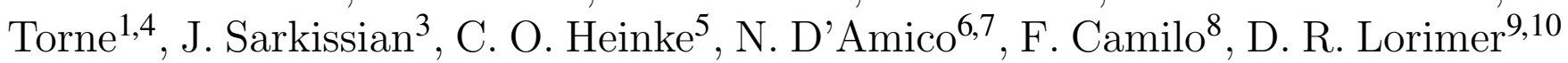 \\ and A. G. Lyne ${ }^{2}$ \\ ${ }^{1}$ Max-Planck-Institut für Radioastronomie, Auf dem Hügel 69, D-53121 Bonn, Germany \\ ${ }^{2}$ Jodrell Bank Centre for Astrophysics, School of Physics and Astronomy, The University of Manchester, Manchester M13 9PL, UK \\ ${ }^{3}$ CSIRO Astronomy and Space Science, Australia Telescope National Facility, Box 76, Epping, NSW 1710, Australia \\ ${ }^{4}$ Instituto de Radioastronomía Milimétrica, Avda. Divina Pastora 7, Núcleo Central, 18012, Granada, Spain \\ ${ }^{5}$ Physics Department, University of Alberta, 4-183 CCIS, Edmonton, AB T6G 2G7, Canada \\ ${ }^{6}$ Osservatorio Astronomico di Cagliari, INAF, via della Scienza 5, I-09047 Selargius (CA), Italy \\ ${ }^{7}$ Dipartimento di Fisica, Università degli Studi di Cagliari, SP Monserrato-Sestu km 0,7, 90042 Monserrato (CA), Italy \\ ${ }^{8}$ Square Kilometre Array South Africa, Pinelands, 7405, South Africa \\ ${ }^{9}$ Department of Physics and Astronomy, West Virginia University, P.O. Box 6315, Morgantown, WV 26506, USA \\ ${ }^{10}$ Center for Gravitational Waves and Cosmology, Chestnut Ridge Research Building, Morgantown, WV 26505, USA
}

Accepted XXX. Received YYY; in original form ZZZ

\begin{abstract}
This paper is the second in a series where we report the results of the long-term timing of the millisecond pulsars (MSPs) in 47 Tucanae with the Parkes $64-\mathrm{m}$ radio telescope. We obtain improved timing parameters that provide additional information for studies of the cluster dynamics: a) the pulsar proper motions yield an estimate of the proper motion of the cluster as a whole $\left(\mu_{\alpha}=5.00 \pm 0.14 \mathrm{mas} \mathrm{yr}^{-1}, \mu_{\delta}=\right.$

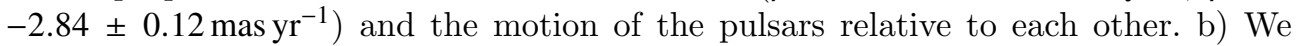
measure the second spin-period derivatives caused by the change of the pulsar line-ofsight accelerations; 47 Tuc H, U and possibly J are being affected by nearby objects. c) For ten binary systems we now measure changes in the orbital period caused by their acceleration in the gravitational field of the cluster. From all these measurements, we derive a cluster distance no smaller than $\sim 4.69 \mathrm{kpc}$ and show that the characteristics of these MSPs are very similar to their counterparts in the Galactic disk. We find no evidence in favour of an intermediate mass black hole at the centre of the cluster. Finally, we describe the orbital behaviour of four "black widow" systems. Two of them, 47 Tuc J and O, exhibit orbital variability similar to that observed in other such systems, while for 47 Tuc I and R the orbits seem to be remarkably stable. It appears, therefore, that not all "black widows" have unpredictable orbital behaviour.
\end{abstract}

Key words: (stars:)binaries: general - pulsars: individual:PSR J0024-7203C to J0024-7204ab - globular clusters: individual:47 Tucanae

\section{INTRODUCTION}

The year 2017 marks the $30^{\text {th }}$ anniversary of the discovery of the first radio pulsar in a globular cluster (GC), PSR B1821-24 (Lyne et al. 1987). Since then these clusters have been shown to be extremely prolific millisecond pulsar (MSP) factories; the total number of pulsars discovered in

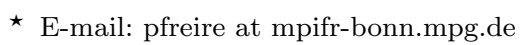

GCs is now ${ }^{1} 149$, in a total of 28 Galactic globular clusters (for a recent review, see Freire 2013), the vast majority of which are MSPs. This makes the GC pulsar population very different from that of the Galactic disk. The total number of radio pulsars in the Galactic GCs is probably of the order of a few thousand (Turk \& Lorimer 2013), most of which have not yet been found because of the insufficient sensitivity and sky coverage of extant radio telescopes. Their discovery will

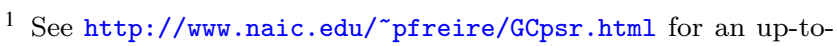
date list.
} 
Table 1. References for the radio work on the pulsars in the globular cluster 47 Tucanae (NGC 104). Pulsars A, B and K do not exist. In boldface, we highlight the five pulsars discovered since 2000. The asterisks indicate the phase-coherent timing solutions determined since 2003. In the second column, we describe the type of system: WD binary implies that the companion is a white dwarf star, BW and RB imply black widow and redback systems respectively, with an (e) indicating our detection of radio eclipses. In the third column we indicate the reference of the discovery, in the fourth column the reference with the first orbital solution precise enough to predict the orbital phase for the whole data set. For instance, Camilo et al. (2000) published approximate orbital parameters for pulsars $\mathrm{P}$, R and $\mathrm{W}$, but these were not precise enough to predict the orbital phase many days in advance. All known binary systems now have well determined orbital parameters. In the penultimate column we indicate the first publication with a timing solution and in the last we list which timing solutions are presented in this work (and whether they are updates or new solutions).

\begin{tabular}{|c|c|c|c|c|c|}
\hline $\begin{array}{l}\text { Pulsar } \\
47 \text { Tuc }\end{array}$ & Type & $\begin{array}{l}\text { Discovery } \\
\text { (Reference) }\end{array}$ & $\begin{array}{c}\text { Orbit } \\
\text { (Reference) }\end{array}$ & $\begin{array}{c}\text { First Timing Solution } \\
\text { (Reference) }\end{array}$ & $\begin{array}{c}\text { Timing } \\
\text { (This work) }\end{array}$ \\
\hline $\mathrm{C}$ & isolated & Manchester et al. (1990) & - & Robinson et al. (1995) & update \\
\hline $\mathrm{D}$ & isolated & Manchester et al. (1991) & - & Robinson et al. (1995) & update \\
\hline $\mathrm{E}$ & WD binary & Manchester et al. (1991) & Robinson et al. (1995) & Freire et al. (2001b) & update \\
\hline $\mathrm{F}$ & isolated & Manchester et al. (1991) & - & Freire et al. (2001b) & update \\
\hline G & isolated & Manchester et al. (1991) & - & Freire et al. (2001b) & update \\
\hline $\mathrm{H}$ & WD binary & Manchester et al. (1991) & Camilo et al. (2000) & Freire et al. (2001b) & update \\
\hline I & BW & Manchester et al. (1991) & Robinson et al. (1995) & Freire et al. (2001b) & update \\
\hline $\mathrm{J}$ & $\mathrm{BW}(\mathrm{e})$ & Manchester et al. (1991) & Robinson et al. (1995) & Camilo et al. (2000) & update \\
\hline $\mathrm{L}$ & isolated & Manchester et al. (1991) & - & Freire et al. (2001b) & update \\
\hline M & isolated & Manchester et al. (1991) & - & Freire et al. (2001b) & update \\
\hline $\mathrm{N}$ & isolated & Robinson et al. (1995) & - & Freire et al. (2001b) & update \\
\hline $\mathrm{O}$ & $\mathrm{BW}(\mathrm{e})$ & Camilo et al. (2000) & Camilo et al. (2000) & Freire et al. (2001b) & update \\
\hline $\mathrm{P}$ & BW & Camilo et al. (2000) & Ridolfi et al. (2016) & & $\ldots$ \\
\hline $\mathrm{Q}$ & WD binary & Camilo et al. (2000) & Camilo et al. (2000) & Freire et al. (2001b) & update \\
\hline $\mathrm{R} *$ & BW (e) & Camilo et al. (2000) & This work & This work & new \\
\hline $\mathrm{S}$ & WD binary & Camilo et al. (2000) & Freire et al. (2001a) & Freire et al. (2003) & update \\
\hline $\mathrm{T}$ & WD binary & Camilo et al. (2000) & Freire et al. (2001a) & Freire et al. (2001b) & update \\
\hline $\mathrm{U}$ & WD binary & Camilo et al. (2000) & Camilo et al. (2000) & Freire et al. (2001b) & update \\
\hline $\mathrm{V}$ & RB (e) & Camilo et al. (2000) & Ridolfi et al. (2016) & $\ldots$ & $\ldots$ \\
\hline $\mathrm{W} *$ & RB (e) & Camilo et al. (2000) & Ridolfi et al. (2016) & Ridolfi et al. (2016) & no \\
\hline $\mathbf{X}^{*}$ & WD binary & This work & Ridolfi et al. (2016) & Ridolfi et al. (2016) & no \\
\hline $\mathbf{Y}^{*}$ & WD binary & This work & This work & This work & new \\
\hline $\mathbf{Z} *$ & isolated & Knight (2007) & - & This work & new \\
\hline aa * & isolated & Pan et al. (2016) & - & Freire \& Ridolfi (2017) & no \\
\hline $\mathbf{a b} *$ & isolated & Pan et al. (2016) & - & Pan et al. (2016) & update \\
\hline
\end{tabular}

probably have to wait for the construction of the Five hundred meter Aperture Spherical Telescope (Smits et al. 2009) or the Square Kilometer Array (SKA, Hessels et al. 2015).

The globular cluster 47 Tucanae (also known as NGC 104, henceforth 47 Tuc) has a total of 25 known radio pulsars, second only to the GC Terzan 5, which has 37 known pulsars (see e.g., Ransom et al. 2005; Hessels et al. 2006, Cadelano et al. in prep.). All pulsars in 47 Tuc have spin periods smaller than $8 \mathrm{~ms}$; of these, 15 are in binary systems (see Table 1). These discoveries and subsequent timing (see also Table 1 for the references) have enabled unprecedented studies of stellar evolution in GCs (Rasio et al. 2000), studies of cluster dynamics (Freire et al. 2003) and even the discovery of ionised gas in the cluster, the first ever detection of any sort of interstellar medium within a GC (Freire et al. 2001c). In addition, all the pulsars with well-determined positions have been identified at X-ray wavelengths (Grindlay et al. 2001; Grindlay et al. 2002; Heinke et al. 2005; Bogdanov et al. 2006; Ridolfi et al. 2016, Bhattacharya et al., submitted) and at least 6 companion objects have been identified at optical wavelengths (Edmonds et al. 2001, 2002; Rivera-Sandoval et al. 2015; Cadelano et al. 2015).

In the first paper from this series (Ridolfi et al. 2016, henceforth Paper I) we described the motivation, observations and data processing of the long-term radio monitoring of the radio pulsars in 47 Tuc with the $64-\mathrm{m}$ Parkes radio telescope. That paper focused on one of the objectives of the long-term timing, the characterization of the elusive binary pulsars 47 Tuc $\mathrm{P}, \mathrm{V}, \mathrm{W}$ and $\mathrm{X}$; for two of those systems
(47 Tuc $\mathrm{W}$ and $\mathrm{X}$ ) it was possible to derive phase-coherent timing solutions. Of these systems, 47 Tuc $\mathrm{X}$ is especially interesting - it is a binary with an extremely low eccentricity that lies well outside the central regions of the cluster.

In this paper, the second in the series, we present upto-date timing solutions for 20 of the 25 MSPs in 47 Tuc, which include all data from the Analogue Filterbank (AFB, see Section 2). The bulk of the paper is a discussion of some of the implications of the 23 known timing solutions (we also use the solutions for 47 Tuc $\mathrm{W}$ and $\mathrm{X}$ derived in Paper I, and the timing solution for 47 Tuc aa, to be presented in Freire \& Ridolfi 2017, in prep.).

We discuss the proper motions in Section 3, the new "jerk" measurements in Section 4 and in Section 5 we discuss the variation of the orbital periods for a set of ten binary systems. This includes estimates of the line-of-sight component of the accelerations of the systems in the gravitational field of the cluster, which can be used to determine their true spin-down parameters. In Section 6, we discuss the longterm orbital behaviour of the four "black widow" systems ${ }^{2}$ with known timing solutions in 47 Tuc, and in Section 7 we present the detection of the rate of advance of periastron in three MSP-WD binaries and a refined measurement of the mass of the 47 Tuc $\mathrm{H}$ binary system. We discuss some of the implications of our results in Section 8 and summarise our findings in Section 9.

2 See Roberts (2013) for a review of "black widow" and "redback" pulsar binary systems 

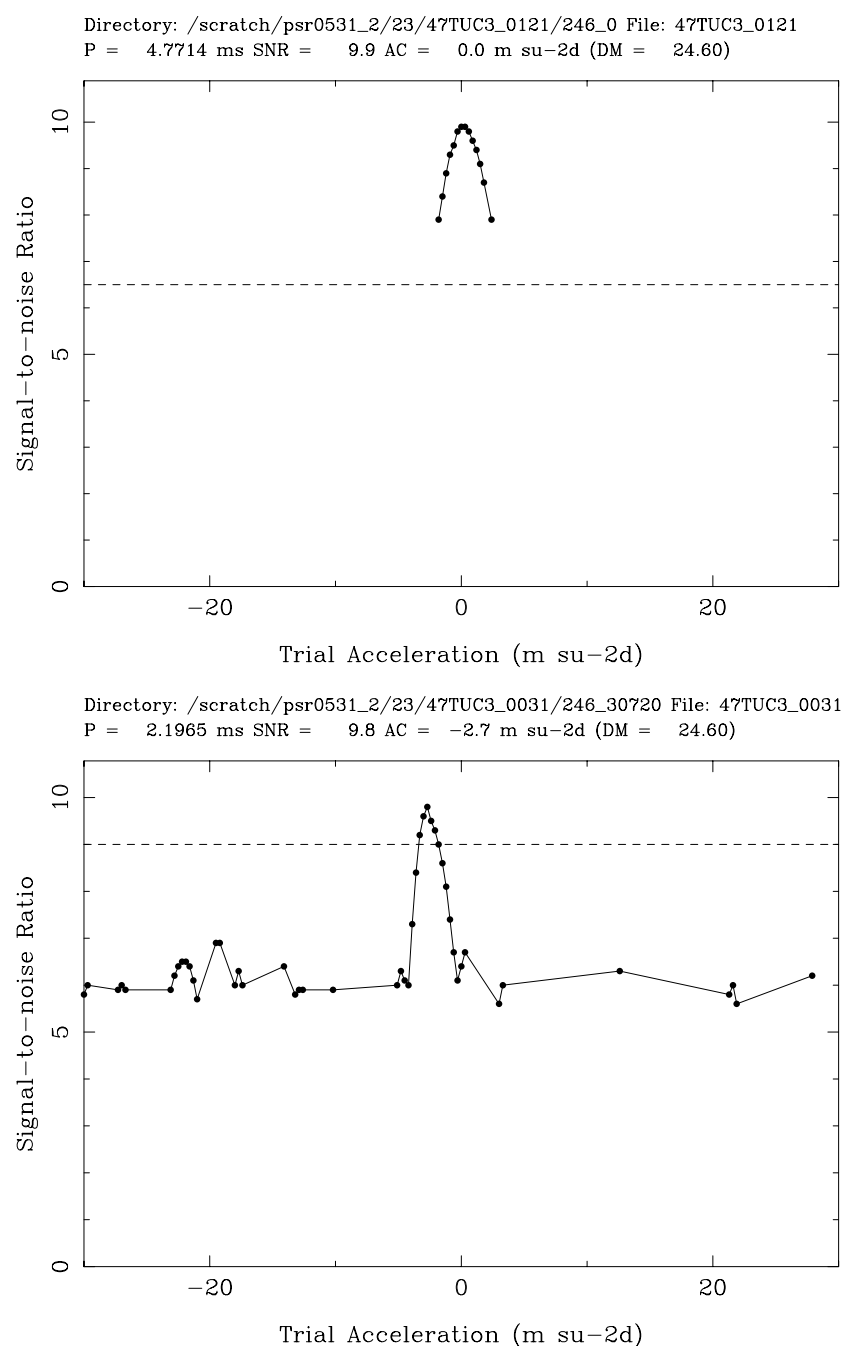

Figure 1. Discovery plots for 47 Tuc X (top) and 47 Tuc Y (bottom) from the AS23 survey, described by Camilo et al. (2000). Each plot shows how the signal-to-noise ratio varies as a function of trial acceleration. These candidates could not be confirmed within the scope of AS23; they were later confirmed by the AS25 survey (see text).

All of these results benefit greatly from the much larger timing baseline presented in this paper in comparison with that in previous publications. They provide information that will be used to produce improved dynamical models of the cluster, a better model of the intracluster gas distribution, and will provide input for stellar evolution models, all of these being strong motivations for the long-term timing of the cluster.

\subsection{Cluster parameters}

In the analysis presented below, we benefit greatly from the new studies of Hubble Space Telescope (HST) data that have become available since our last study of the cluster potential (Freire et al. 2003), these provide much more precise cluster parameters and in some cases entirely new information. McLaughlin et al. (2006) placed the centre of 47 Tuc at right ascension $\alpha=00^{\mathrm{h}} 24^{\mathrm{m}} 5^{\mathrm{s}} .67$ and declination $\delta=-72^{\circ} 04^{\prime} 52^{\prime \prime} 62$; they also measured the angular core radius: $\theta_{c}=0.347^{\prime}$. A newly available measurement, which will be of great importance for this work, is the 1-D proper motion dispersion for stars at the cluster centre obtained from differential HST astrometry: $\sigma_{\mu, 0}=0.573 \mathrm{mas} \mathrm{yr}^{-1}$ (Watkins et al. 2015a).

From Eq. 1-34 in Spitzer (1987), which is accurate to $\sim 0.5 \%$ for clusters where the tidal radius $r_{t}$ is much larger than the core radius $r_{c}$ (such as 47 Tuc, where $c=\log _{10}\left(r_{t} / r_{c}\right)=2.07$, see Harris 1996), we derive the following expression for the central density:

$\rho(0)=\frac{9 \sigma_{\mu, 0}^{2}}{4 \pi G \theta_{c}^{2}}$

where we have replaced the spectroscopic radial velocity (RV) dispersion $\sigma_{0}$ with $\sigma_{\mu, 0} d$ (where $d$ is the distance to 47 Tuc) and the core radius $r_{c}$ with $\theta_{c} d$. The distance terms then cancel out; this means that the central density can be determined solely from the aforementioned angular measurements, independently of $d$. For the $\sigma_{\mu, 0}$ and $\theta_{c}$ of 47 Tuc Eq. 1 yields $\rho(0)=1.20 \times 10^{5} M_{\odot} \mathrm{pc}^{-3}$.

For $d$, we use $4.69 \mathrm{kpc}$ (Woodley et al. 2012). Other recent assessments place the cluster at very similar distances; for instance, using the (relatively well-trusted) white dwarf (WD) cooling track model, Hansen et al. (2013) derived $d=4.6 \pm 0.2 \mathrm{kpc}$. Using other methods, like the selfconsistent isochrone fits to colour-magnitude diagrams and the eclipsing binary star V69, Brogaard et al. (2017) derived a slightly smaller $d=4.4 \pm 0.2 \mathrm{kpc}$. Averaging several recent measurements, Bogdanov et al. (2016) obtained $d=4.53_{-0.04}^{+0.08} \mathrm{kpc}$.

However, not all distance estimates match: By comparing their measurement of $\sigma_{\mu, 0}$ to their best estimate of $\sigma_{0}$, Watkins et al. (2015b) derived a kinematic $d=4.15 \pm$ $0.08 \mathrm{kpc}$, consistent with the earlier estimate of McLaughlin et al. $(2006), d=4.02 \pm 0.35 \mathrm{kpc}$.

A possible explanation of this discrepancy is that the $\sigma_{0}$ measurements (generally close to $11 \mathrm{~km} \mathrm{~s}^{-1}$ ), are biased towards smaller values. Likely reasons for this were discussed in detail in Bogdanov et al. (2016). Briefly, those authors pointed out that the RV measurements used for comparison were intended to be of single stars, however HST images show that a number of the targeted "stars" actually comprise more than one star of similar brightness. The RV measurements of combined stars tend to be closer to the cluster mean than single stars, so this has the effect of reducing the inferred velocity dispersion and the resultant $d$. Indeed, when Watkins et al. (2015b) included a larger sample of RV measurements that extend further out from the core of 47 Tuc to compare with their proper motions, they found $d=4.61_{-0.07}^{+0.06} \mathrm{kpc}$ (see their Appendix A, Fig. 9), consistent with the larger distances mentioned above.

This issue is crucial for the interpretation of our results. As we will see in Sections 4 and 5, our results also favour this large distance, rather than the smaller kinematic distance estimates. This issue is also crucial, as we shall see, for addressing the question of the presence of an intermediate mass black hole in the centre of 47 Tucanae, which has been repeatedly raised in the literature. 

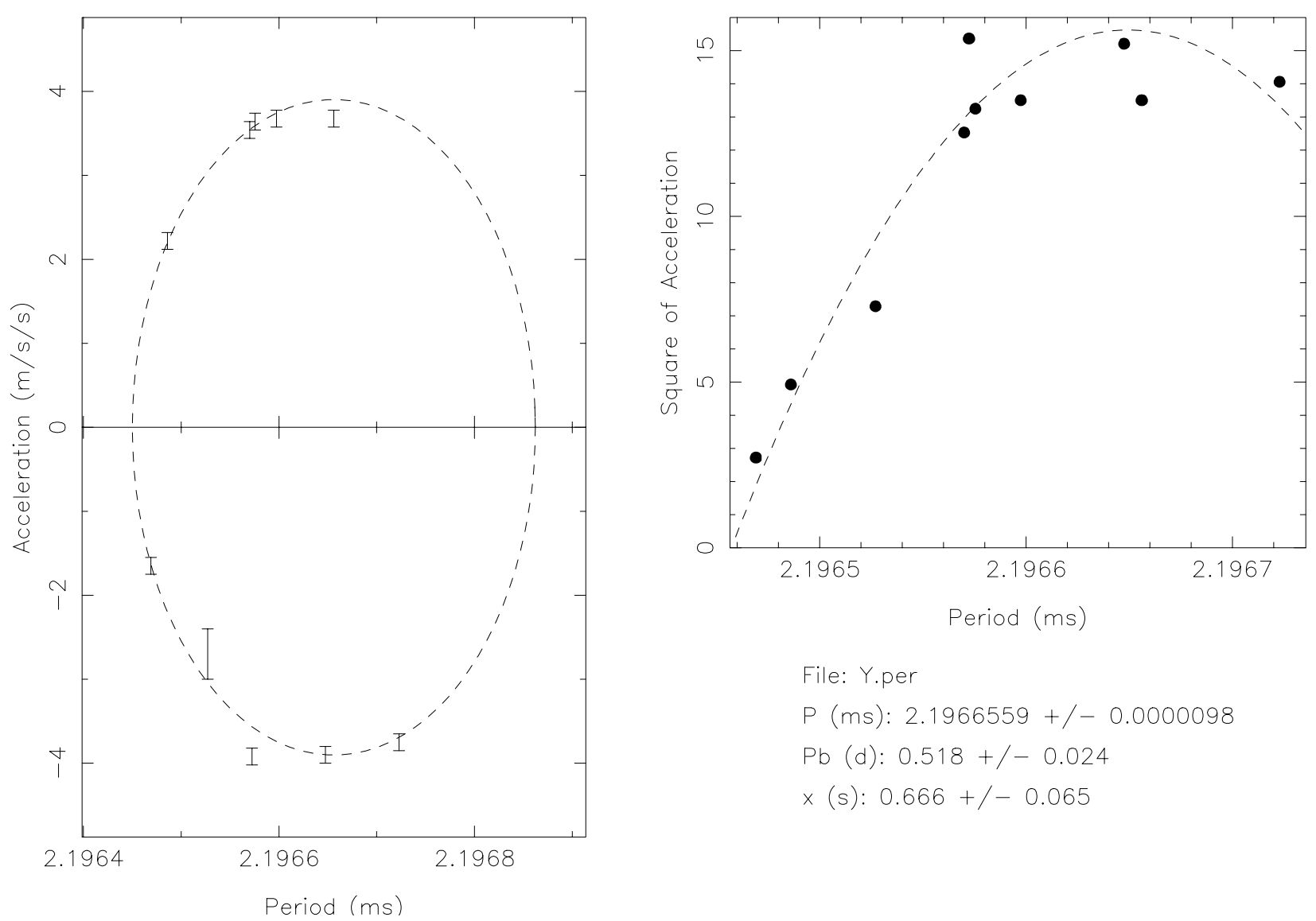

Figure 2. For 47 Tuc Y, the AS25 detections were sufficient for the determination of the orbit using the CIRCORBIT routine, described by Freire et al. (2001a). In the right panel, a parabola was fit to the squares of the acceleration as a function of spin period. This is translated (in the left panel) to a best-fitting ellipse (dashed) to the observed accelerations as a function of barycentric spin period, represented by the vertical error bars. This ellipse corresponds to the spin and orbital parameters listed in the bottom right.

\section{DATA REDUCTION}

The data analysed here were taken with the Australian 64$\mathrm{m}$ Parkes radio telescope. The observations, receivers and signal-processing systems were described in Paper I. Two filterbank systems were used: the low-resolution $2 \times 96 \times 3$ $\mathrm{MHz}$ analogue filterbank and the $2 \times 512 \times 0.5 \mathrm{MHz}$ highresolution analogue filterbanks, henceforth the $\mathrm{AAFB}$ and hAFB, respectively.

\subsection{Discovery of two millisecond pulsars}

Before moving to the bulk of the paper, we report on the discovery of two MSPs, 47 Tuc X and Y. These pulsars, with periods of 4.771 and $2.196 \mathrm{~ms}$ (see Fig. 1) were initially found in the survey described by Camilo et al. (2000), but could only be confirmed in a deeper survey briefly described in Freire et al. (2001a). This used the same software (the SIGPROC acceleration search routines ${ }^{3}$ ) and data (the lAFB data) used in Camilo et al. (2000). The only difference was that instead of searching 17.5-minute segments of data, each containing $2^{23} 125$ - $\mu$ s-long time samples, it used 70 -minute segments, each containing $2^{25}$ time samples; for this reason

\footnotetext{
3 http://sigproc.sourceforge.net/
}

we designate the former AS23 (where AS means "accelerated search") and the latter AS25. Because of computing limitations, AS25 only covered accelerations from -5 to $5 \mathrm{~m} \mathrm{~s}^{-2}$ in steps of $0.02 \mathrm{~m} \mathrm{~s}^{-2}$, while AS23 covered accelerations from -30 to $30 \mathrm{~m} \mathrm{~s}^{-2}$ in steps of $0.3 \mathrm{~m} \mathrm{~s}^{-2}$. In the case of sources with steady flux density, AS25 has twice the sensitivity of AS23 for pulsars with small accelerations.

Some of the earlier results of AS25 were reported in Freire et al. (2001a): 47 Tuc $\mathrm{T}$ and $\mathrm{S}$ (which are always within its acceleration range) were detected enough times to allow the determination of their orbits using the new orbital determination method presented in that paper. Later, 47 Tuc $\mathrm{X}$ and $\mathrm{Y}$ were confirmed by this survey. These two pulsars were also independently discovered in a parallel search by one of us, N. D'Amico, in Bologna, Italy. Their existence was mentioned for the first time in Lorimer et al. (2003).

\subsection{Determination of orbits and timing solutions}

For 47 Tuc Y, the AS25 detections were numerous enough to determine the orbit using the period - acceleration method of Freire et al. (2001a) (see Fig. 2). The orbit is nearly circular and has a period of 12.52 hours and, assuming a pulsar mass of $1.4 \mathrm{M}_{\odot}$, a minimum companion mass of $0.141 \mathrm{M}_{\odot}$. Its parameters were then refined in three stages: 1) finding 

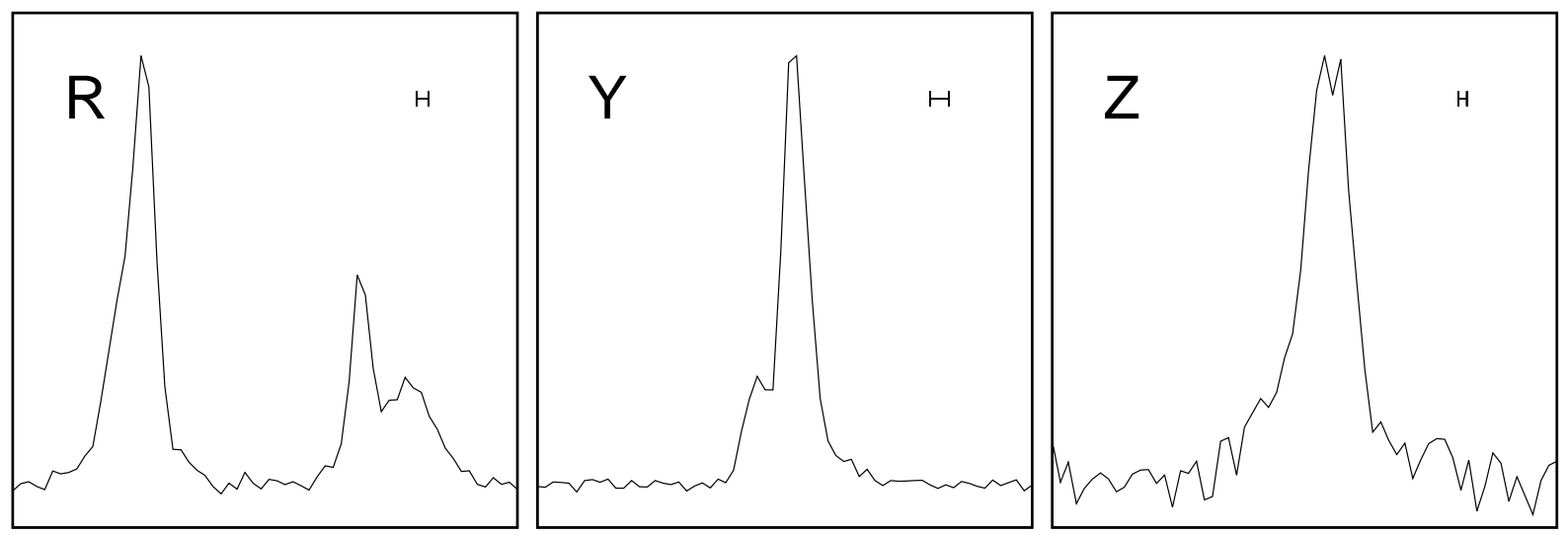

Figure 3. Full-cycle pulse profiles of 47 Tuc R, Y and Z. The horizontal error bars display the time resolution of the hAFB data.

the correct orbit count using the method described in Paper I (i.e., searching for an orbital period that is the common integer sub-multiple of all differences between times of passage through ascending node), 2) by fitting an orbital model to the variation of the spin period versus time, and 3) fitting pulse times of arrival (ToAs) as a function of time using the TEMPO pulsar timing software ${ }^{4}$; the ToAs for this pulsar were derived with the pulse profile presented in Fig 3. For 47 Tuc X, a more sophisticated and thorough search of the hAFB data was necessary for determining its orbit and is reported in Paper I, its pulse profile is also presented there.

After 2003, the AS23 and AS25 searches were extended to all the hAFB data then available. This resulted in two extra detections of 47 Tuc R, an eclipsing black widow with a minimum companion mass of only $0.0264 \mathrm{M}_{\odot}$ (assuming $\left.M_{p}=1.4 \mathrm{M}_{\odot}\right)$ and at the time the binary pulsar with the shortest orbital period known (96 minutes, Camilo et al. 2000). We then determined a precise orbital period for this system using (again) the orbital count technique described in Paper I. Its pulse profile, based on hAFB data, is displayed in Fig. 3. This represents a substantial improvement over the previous profile based on lAFB data in Camilo et al. (2000).

More recently, and using the full hAFB data set, we have been able to detect the isolated MSP 47 Tuc Z (discovered by Knight 2007) enough times to derive a good preliminary ephemeris. Its pulse profile is also displayed in Fig. 3.

For all these pulsars (R, Y and Z), the subsequent processing was the same. All data were folded using their preliminary ephemerides. This increased the number of detections greatly and led to the determination of even better ephemerides, which in turn allowed even more detections. This iterative process eventually allowed the determination of phase-coherent timing solutions of these pulsars, which are presented here for the first time.

Because the timing solutions of 47 Tuc $\mathrm{R}$ and $\mathrm{Y}$ were well determined in 2006, both were included in the group of 19 MSPs for which X-ray emission was detected with the Chandra X-ray observatory in Bogdanov et al. (2006). For 47 Tuc Z, aa and ab, the timing solutions and resulting precise positions were only determined in 2016; their Chandra X-ray analysis is presented in Bhattacharya et al. 2017 (submitted). The pulsars 47 Tuc $\mathrm{R}$ and $\mathrm{Y}$ have also been well studied at optical wavelengths. The WD companion of 47 Tuc Y is clearly detected, and it is the second brightest WD after the WD companion to 47 Tuc U (Rivera-Sandoval et al. 2015; Cadelano et al. 2015).

\subsection{Updated timing solutions}

For this work, all the extant AFB data were de-dispersed and folded anew using the DSPSR routine (van Straten \& Bailes 2011) with the best previous ephemerides for 20 MSPs. The resulting pulse profiles were then cross-correlated with a lownoise profile, normally derived from the best detection(s) of each pulsar, using the method described in Taylor (1992) and implemented in the PSRCHIVE software (Hotan et al. 2004; van Straten et al. 2012).

This resulted in topocentric ToAs which were then analysed with TEMPO, where we used the Jet Propulsion Laboratory (JPL) DE/LE 421 Solar system ephemeris (Folkner et al. 2009) to subtract the effect of the motion of the radio telescope relative to the barycentre of the Solar System.

The timing solutions of the isolated pulsars are presented in Table 2, those of the MSP-WD systems in Table 3 and the solutions of four black widow systems in Table 4 . The TEMPO ephemerides are available online ${ }^{5}$. These solutions describe the data well, with no trends detectable in the ToA residuals, except for slight delays near eclipse phase for some of the eclipsing pulsars (Freire et al. 2003); these are not taken into account in the solutions. The uncertainties presented in all these tables are 1- $\sigma(68 \%)$ confidence limits, and were derived using a Monte-Carlo bootstrap routine implemented in TEMPO. In all solutions, we fixed a parallax that corresponds to the assumed distance to 47 Tuc, $4.69 \mathrm{kpc}$ (Woodley et al. 2012).

\section{PROPER MOTIONS}

In Fig. 4 we display the angular offsets of 22 pulsars in the sky relative to the centre of the cluster. Their numerical values are presented in Tables 2 to 4 . The figure does not display 47 Tuc $\mathrm{X}$, which is at a distance of $\sim 3.8^{\prime}$ from the centre of the cluster (Paper I). All other pulsars (including 
Table 2. Timing parameters for nine of the ten isolated pulsars in 47 Tuc, as obtained from fitting the observed ToAs with TEMPO. For all the pulsars in this and the following tables, the reference epoch is MJD 51600; the 1- $\sigma$ uncertainties are calculated using a Monte-Carlo bootstrap routine implemented in TEMPO. A fixed parallax value of 0.2132 mas was assumed; the time units are TDB; the adopted terrestrial time standard is UTC(NIST); the Solar System ephemeris used is JPL DE421.

\begin{tabular}{|c|c|c|c|c|}
\hline Pulsar & 47 Tuc $\mathrm{C}$ & 47 Tuc D & 47 Tuc $\mathrm{F}$ & 47 Tuc $\mathrm{G}$ \\
\hline Right Ascension, $\alpha(\mathrm{J} 2000) \ldots \ldots$ & $00: 23: 50.3546(1)$ & $00: 24: 13.88092(6)$ & $00: 24: 03.85547(10)$ & $00: 24: 07.9603(1)$ \\
\hline Declination, $\delta(\mathrm{J} 2000) \ldots \ldots \ldots \ldots \ldots \ldots \ldots \ldots \ldots \ldots \ldots \ldots$ & $-72: 04: 31.5048(4)$ & $-72: 04: 43.8524(2)$ & $-72: 04: 42.8183(2)$ & $-72: 04: 39.7030(5)$ \\
\hline Proper Motion in $\alpha, \mu_{\alpha}\left(\right.$ mas $\left.\mathrm{yr}^{-1}\right) \ldots \ldots \ldots \ldots$ & $5.2(1)$ & $4.24(7)$ & $4.52(8)$ & $4.5(1)$ \\
\hline Proper Motion in $\delta, \mu_{\delta}\left({\left.\text { mas } \mathrm{yr}^{-1}\right)}\right.$. & $-3.1(1)$ & $-2.24(5)$ & $-2.50(5)$ & $-2.9(1)$ \\
\hline Spin Frequency, $f(\mathrm{~Hz}) \quad \ldots \ldots \ldots \ldots \ldots \ldots \ldots$ & $173.708218965958(4)$ & $186.651669856731(3)$ & $381.158663656311(5)$ & $247.501525096385(8)$ \\
\hline First Spin Frequency derivative, $\dot{f}\left(10^{-15} \mathrm{~Hz} \mathrm{~s}^{-1}\right) \ldots$ & $1.50421(6)$ & $0.11922(3)$ & $-9.3711(1)$ & $2.5825(1)$ \\
\hline Second Spin Frequency derivative, $\ddot{f}\left(10^{-27} \mathrm{~Hz} \mathrm{~s}^{-2}\right)$ & $1.3(4)$ & $-1.2(2)$ & $6.8(7)$ & $6.0(9)$ \\
\hline Start of Timing Data (MJD) $\ldots \ldots \ldots \ldots \ldots \ldots$ & 47857.439 & 47716.842 & 48494.790 & 48600.489 \\
\hline End of Timing Data (MJD) $\ldots \ldots \ldots \ldots \ldots \ldots$ & 56508.971 & 56508.976 & 56466.879 & 56466.879 \\
\hline Dispersion Measure, $\mathrm{DM}\left(\mathrm{pc} \mathrm{cm}^{-3}\right) \ldots \ldots \ldots \ldots$ & $24.600(4)$ & $24.732(3)$ & $24.382(5)$ & $24.436(4)$ \\
\hline Number of ToAs ................ & 6225 & 3607 & 1785 & 594 \\
\hline Residuals $\operatorname{RMS}(\mu \mathrm{s}) \ldots \ldots \ldots \ldots \ldots \ldots \ldots \ldots \ldots \ldots$ & 12.33 & 8.74 & 7.83 & 11.25 \\
\hline \multicolumn{5}{|c|}{ Derived Parameters } \\
\hline Angular offset from centre in $\alpha, \theta_{\alpha}(\operatorname{arcmin}) \ldots \ldots$ & -1.1784 & +0.6316 & -0.1396 & +0.1762 \\
\hline Angular offset from centre in $\delta, \theta_{\delta}(\operatorname{arcmin}) \ldots \ldots$ & +0.3520 & +0.1460 & +0.1634 & +0.2151 \\
\hline Total angular offset from centre, $\theta_{\perp}(\operatorname{arcmin}) \ldots$ & 1.2298 & 0.6483 & 0.2149 & 0.2781 \\
\hline Total angular offset from centre, $\theta_{\perp}$ (core radii) $\ldots$ & 3.5442 & 1.8683 & 0.6194 & 0.8014 \\
\hline Projected distance from centre, $r_{\perp}(\mathrm{pc}) \ldots \ldots \ldots$ & 1.6778 & 0.8845 & 0.2932 & 0.3794 \\
\hline 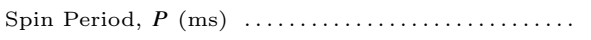 & $5.7567799955164(1)$ & $5.35757328486572(7)$ & $2.62357935251262(3)$ & $4.0403791435651(1)$ \\
\hline First Spin Period derivative, $\dot{P}_{\mathrm{obs}}\left(10^{-21} \mathrm{~s} \mathrm{~s}^{-1}\right) \ldots$ & $-49.850(2)$ & $-3.4219(9)$ & $64.5031(7)$ & $-42.159(2)$ \\
\hline Line-of-sight jerk, $\dot{a}_{\ell}\left(10^{-21} \mathrm{~m} \mathrm{~s}^{-3}\right) \ldots \ldots \ldots \ldots \ldots$ & $-2.3(7)$ & $1.98(26)$ & $-5.3(5)$ & $-7.3(1.1)$ \\
\hline
\end{tabular}

Table 2 - continued

\begin{tabular}{|c|c|c|c|c|c|}
\hline Pulsar & 47 Tuc L & 47 Tuc M & 47 Tuc $\mathrm{N}$ & 47 Tuc $\mathrm{Z}$ & 47 Tuc ab \\
\hline Right Ascension, $\alpha(\mathrm{J} 2000) \ldots \ldots \ldots \ldots \ldots \ldots$ & $00: 24: 03.7721(3)$ & $00: 23: 54.4899(3)$ & $00: 24: 09.1880(2)$ & $00: 24: 06.041(2)$ & $00: 24: 08.1615(5)$ \\
\hline Declination, $\delta(\mathrm{J} 2000) \ldots \ldots \ldots \ldots \ldots \ldots \ldots \ldots \ldots$ & $-72: 04: 56.923(2)$ & $-72: 05: 30.756(2)$ & $-72: 04: 28.8907(7)$ & $-72: 05: 01.480(6)$ & $-72: 04: 47.602(2)$ \\
\hline Proper Motion in $\alpha, \mu_{\alpha}\left(\operatorname{mas} \mathrm{yr}^{-1}\right) \ldots \ldots \ldots \ldots$ & $4.4(2)$ & $5.0(3)$ & $6.3(2)$ & $4(2)$ & $4.2(6)$ \\
\hline Proper Motion in $\delta, \mu_{\delta}\left(\operatorname{mas}_{\mathrm{yr}^{-1}}\right) \ldots \ldots \ldots \ldots$ & $-2.4(2)$ & $-2.0(4)$ & $-2.8(2)$ & $1(2)$ & $-2.9(5)$ \\
\hline Spin Frequency, $f(\mathrm{~Hz}) \ldots \ldots \ldots \ldots \ldots \ldots \ldots$ & $230.08774629142(2)$ & $271.98722878874(2)$ & $327.44431861739(1)$ & $219.5656060346(1)$ & $269.93179806134(4)$ \\
\hline First Spin Frequency derivative, $\dot{f}\left(10^{-15} \mathrm{~Hz} \mathrm{~s}^{-1}\right) \ldots$ & $6.4611(2)$ & $2.8421(4)$ & $2.3435(2)$ & $0.219(3)$ & $-0.7155(6)$ \\
\hline Second Spin Frequency derivative, $\ddot{f}\left(10^{-27} \mathrm{~Hz} \mathrm{~s}^{-2}\right)$ & $-1.3(1.3)$ & $7(2)$ & $-9(2)$ & $8(25)$ & $-8(3)$ \\
\hline 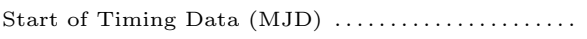 & 50686.683 & 48491.694 & 48515.534 & 51003.792 & 51000.785 \\
\hline End of Timing Data (MJD) $\ldots \ldots \ldots \ldots \ldots \ldots \ldots$ & 56388.208 & 55526.513 & 55648.110 & 54645.852 & 56388.135 \\
\hline Dispersion Measure, DM $\left(\mathrm{pc} \mathrm{cm}^{-3}\right) \ldots \ldots \ldots \ldots$ & $24.40(1)$ & $24.43(2)$ & $24.574(9)$ & $24.45(4)$ & $24.37(2)$ \\
\hline Number of ToAs $\ldots \ldots \ldots \ldots \ldots \ldots \ldots \ldots \ldots . . . \ldots \ldots$ & 411 & 315 & 436 & 107 & 210 \\
\hline Residuals RMS $(\mu \mathrm{s}) \ldots \ldots \ldots$ & 17.02 & 20.15 & 12.98 & 58.78 & 24.85 \\
\hline \multicolumn{6}{|c|}{ Derived Parameters } \\
\hline Angular offset from centre in $\alpha, \theta_{\alpha}(\operatorname{arcmin}) \ldots \ldots$ & -0.1460 & -0.8594 & +0.2707 & +0.0286 & +0.1917 \\
\hline Angular offset from centre in $\delta, \theta_{\delta}(\operatorname{arcmin}) \ldots \ldots$ & -0.0719 & -0.6354 & +0.3955 & -0.1479 & +0.0838 \\
\hline Total angular offset from centre, $\theta_{\perp}(\operatorname{arcmin}) \ldots$ & 0.1627 & 1.0688 & 0.4793 & 0.1506 & 0.2092 \\
\hline Total angular offset from centre, $\theta_{\perp}($ core radii) $\ldots$ & 0.4689 & 3.0801 & 1.3812 & 0.4340 & 0.6028 \\
\hline Projected distance from centre, $r_{\perp}(\mathrm{pc}) \ldots \ldots \ldots$ & 0.2220 & 1.4581 & 0.6539 & 0.2054 & 0.2854 \\
\hline 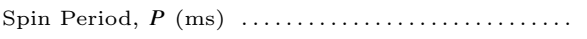 & $4.3461679994616(3)$ & $3.6766432176002(3)$ & $3.0539543462608(1)$ & $4.554447383906(3)$ & $3.7046394947985(5)$ \\
\hline First Spin Period derivative, $\dot{P}_{\mathrm{obs}}\left(10^{-21} \mathrm{~s} \mathrm{~s}^{-1}\right)$ & $-122.0406(10)$ & $-38.418(5)$ & $-21.857(2)$ & $-4.56(1)$ & $9.820(8)$ \\
\hline Line-of-sight jerk, $\dot{a}_{\ell}\left(10^{-21} \mathrm{~m} \mathrm{~s}^{-3}\right) \ldots \ldots \ldots \ldots \ldots$ & $1.7(1.7)$ & $-8.0(2.6)$ & $8.5(1.5)$ & $-11(33)$ & $8.7(3.6)$ \\
\hline
\end{tabular}


Table 3. Timing parameters for seven of the eight MSP-WD binaries in 47 Tuc, as obtained from fitting the observed ToAs with TEMPO. The eighth MSP-WD system, 47 Tuc X, was already studied in detail in Paper I. The orbital models used are DD (Damour \& Deruelle 1985, 1986) and ELL1 (Lange et al. 2001). For the characteristic age, we either present the median or a 2- $\sigma$ lower limit.

\begin{tabular}{|c|c|c|c|c|}
\hline Pulsar & 47 Tuc E & 47 Tuc $\mathrm{H}$ & 47 Tuc $\mathrm{Q}$ & 47 Tuc S \\
\hline Right Ascension, $\alpha(\mathrm{J} 2000) \ldots \ldots \ldots \ldots \ldots \ldots \ldots$ & $00: 24: 11.10528(5)$ & $00: 24: 06.7032(2)$ & $00: 24: 16.4909(1)$ & $00: 24: 03.9794(1)$ \\
\hline 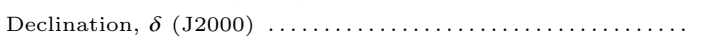 & $-72: 05: 20.1492(2)$ & $-72: 04: 06.8067(6)$ & $-72: 04: 25.1644(6)$ & $-72: 04: 42.3530(4)$ \\
\hline Proper Motion in $\alpha, \mu_{\alpha}\left({\left.\operatorname{mas~} \mathrm{yr}^{-1}\right) \ldots \ldots \ldots \ldots \ldots \ldots \ldots \ldots \ldots \ldots \ldots \ldots \ldots \ldots \ldots}\right.$ & $6.15(3)$ & $5.1(2)$ & $5.2(1)$ & $4.5(1)$ \\
\hline Proper Motion in $\delta, \mu_{\delta}\left({\left.\operatorname{mas~} \mathrm{yr}^{-1}\right) \ldots \ldots \ldots \ldots \ldots \ldots \ldots \ldots \ldots \ldots \ldots \ldots \ldots \ldots \ldots}\right.$ & $-2.35(6)$ & $-2.8(2)$ & $-2.6(1)$ & $-2.5(1)$ \\
\hline 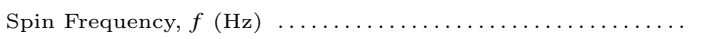 & $282.779107035000(3)$ & $311.49341784423(1)$ & $247.943237418920(9)$ & $353.306209385356(9)$ \\
\hline First Spin Frequency derivative, $\dot{f}\left(10^{-15} \mathrm{~Hz} \mathrm{~s}^{-1}\right) \ldots \ldots \ldots \ldots$ & $-7.87728(4)$ & $0.1775(1)$ & $-2.0907(2)$ & $15.0466(1)$ \\
\hline Second Spin Frequency derivative, $\ddot{f}\left(10^{-27} \mathrm{~Hz} \mathrm{~s}^{-2}\right) \ldots \ldots \ldots$ & $2.9(2)$ & $1.60(2) \times 10^{-25}$ & $7(11) \times 10^{-28}$ & $-7.8(8) \times 10^{-27}$ \\
\hline Start of Timing Data (MJD) $\ldots \ldots \ldots \ldots \ldots \ldots \ldots \ldots \ldots \ldots$ & 48464.854 & 48517.512 & 50689.700 & 50686.683 \\
\hline End of Timing Data (MJD) $\ldots \ldots \ldots \ldots \ldots \ldots \ldots \ldots \ldots \ldots$ & 56508.948 & 56508.972 & 56388.178 & 56466.879 \\
\hline Dispersion Measure, DM ( $\left.\mathrm{pc} \mathrm{cm}^{-3}\right)$ & $24.236(2)$ & $24.369(8)$ & $24.265(4)$ & $24.376(4)$ \\
\hline Number of ToAs $\ldots \ldots \ldots \ldots \ldots \ldots$ & 1812 & 1073 & 697 & 577 \\
\hline 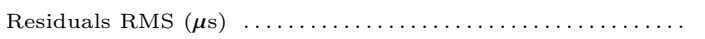 & 6.06 & 17.04 & 12.73 & 9.50 \\
\hline \multicolumn{5}{|c|}{ Binary Parameters } \\
\hline 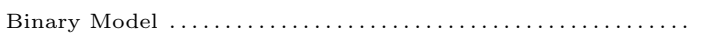 & $\mathrm{DD}$ & $\mathrm{DD}$ & ELL1 & ELL1 \\
\hline Projected Semi-major Axis, $x_{p}$ (lt-s) $\ldots \ldots \ldots \ldots \ldots \ldots \ldots$ & $1.9818427(4)$ & $2.152813(2)$ & $1.4622043(9)$ & $0.7662686(8)$ \\
\hline 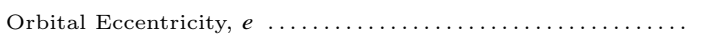 & $3.159(4) \times 10^{-4}$ & $7.0558(1) \times 10^{-2}$ & - & - \\
\hline Longitude of Periastron, $\omega(\operatorname{deg}) \ldots \ldots \ldots \ldots \ldots \ldots \ldots$ & 218.6(1) & $110.603(1)$ & - & - \\
\hline Epoch of passage at Periastron, $T_{0}$ (MJD) $\ldots \ldots \ldots \ldots \ldots$ & $51001.7900(8)$ & $51602.186289(7)$ & - & - \\
\hline First Laplace-Lagrange parameter,$\eta \ldots \ldots \ldots \ldots \ldots \ldots \ldots$ & - & - & $6.2(1) \times 10^{-5}$ & $9.1(3) \times 10^{-5}$ \\
\hline Second Laplace-Lagrange parameter, $\kappa \ldots \ldots \ldots \ldots \ldots \ldots$ & - & - & $-5.1(2) \times 10^{-5}$ & $3.87(2) \times 10^{-4}$ \\
\hline Epoch of passage at Ascending Node, $T_{\text {asc }}(\mathrm{MJD}) \ldots \ldots \ldots$ & - & - & $51600.2842078(2)$ & $51600.6250241(2)$ \\
\hline Rate of periastron advance, $\dot{\omega}(\mathrm{deg} / \mathrm{yr}) \ldots \ldots \ldots \ldots \ldots \ldots$ & $0.090(16)$ & $0.06725(19)$ & - & $0.331(75)$ \\
\hline 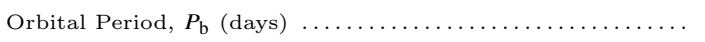 & $2.2568483(9)$ & $2.357696895(10)$ & $1.1890840496(4)$ & $1.2017242354(6)$ \\
\hline Orbital Period derivative, $\dot{P}_{b}\left(10^{-12} \mathrm{~s} \mathrm{~s}^{-1}\right) \ldots \ldots \ldots \ldots \ldots$ & $4.8(2)$ & $-0.7(6)$ & $-1.0(2)$ & $-4.9(4)$ \\
\hline \multicolumn{5}{|c|}{ Derived Parameters } \\
\hline Angular offset from centre in $\alpha, \theta_{\alpha}$ (arcmin) & +0.4179 & +0.0795 & +0.8326 & -0.1301 \\
\hline Angular offset from centre in $\delta, \theta_{\delta}$ (arcmin) & -0.4587 & +0.7636 & +0.4578 & +0.1712 \\
\hline Total angular offset from centre, $\theta_{\perp}(\operatorname{arcmin}) \ldots$ & 0.6205 & 0.7677 & 0.9502 & 0.2150 \\
\hline Total angular offset from centre, $\theta_{\perp}($ core radii) $\ldots \ldots \ldots \ldots$ & 1.7882 & 2.2123 & 2.7383 & 0.6196 \\
\hline Projected distance from centre, $r_{\perp}(\mathrm{pc}) \ldots \ldots \ldots \ldots \ldots \ldots$ & 0.8465 & 1.0473 & 1.2963 & 0.2933 \\
\hline 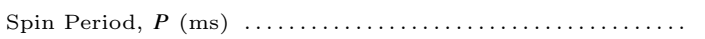 & $3.53632915276243(3)$ & $3.2103407093504(1)$ & $4.0331811845726(2)$ & $2.83040595787912(7)$ \\
\hline First Spin Period derivative, $\dot{P}\left(10^{-21} \mathrm{~s} \mathrm{~s}^{-1}\right) \ldots \ldots \ldots \ldots \ldots$ & $98.5103(5)$ & $-1.830(1)$ & $34.0076(6)$ & $-120.541(1)$ \\
\hline Line of sight acceleration from cluster field, $a_{\ell, \mathrm{GC}}\left(10^{-9} \mathrm{~m} \mathrm{~s}^{-2}\right)$ & $+7.31(32)$ & $-1.0(0.9)$ & $+3.0(0.7)$ & $-14.2(1.1)$ \\
\hline Intrinsic spin period derivative, $\dot{P}_{\text {int }}\left(10^{-21} \mathrm{~s} \mathrm{~s}^{-1}\right) \ldots \ldots \ldots \ldots$ & $11.9(3.7)$ & $9(9)$ & $-6(9)$ & $13(10)$ \\
\hline Characteristic Age, $\tau_{\mathrm{c}}(\mathrm{Gyr}) \ldots \ldots \ldots \ldots \ldots \ldots \ldots \ldots$ & 4.7 & $>1.9$ & $>5.0$ & $>1.3$ \\
\hline Line-of-sight jerk, $\dot{a}_{\ell}\left(10^{-21} \mathrm{~m} \mathrm{~s}^{-3}\right) \ldots \ldots \ldots \ldots \ldots \ldots \ldots \ldots \ldots \ldots$ & $-3.10(26)$ & $-154.5(2.2)$ & $-0.9(1.3)$ & $6.6(6)$ \\
\hline 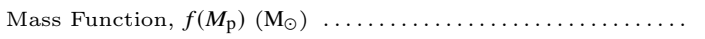 & $0.0016409130(15)$ & $0.001927197(6)$ & $0.002374007(5)$ & $0.0003345154(10)$ \\
\hline Minimum companion mass, $M_{\mathrm{c}, \min }\left(\mathrm{M}_{\odot}\right) \ldots \ldots \ldots \ldots \ldots$ & 0.159 & 0.168 & 0.181 & 0.091 \\
\hline Median companion mass, $M_{\mathrm{c}, \text { med }}\left(\mathrm{M}_{\odot}\right) \ldots \ldots \ldots \ldots \ldots \ldots$ & 0.185 & 0.196 & 0.212 & 0.105 \\
\hline 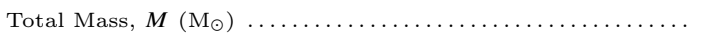 & $2.3(7)$ & $1.665(7)$ & - & $3.1(1.1)$ \\
\hline
\end{tabular}

those with new timing solutions) are well within the distance of 47 Tuc $\mathrm{C}$ to the centre of the cluster, $1.22^{\prime}$. The Parkes $20 \mathrm{~cm}$ beam has a half-power radius of $7^{\prime}$, so this is not a selection effect. As shown by Heinke et al. (2005), the real cause is mass segregation: this close to the centre the relaxation time is much shorter than the age of the cluster, hence, the pulsars are likely to have reached dynamical equilibrium with the stellar population there.

One of the main benefits of long-term timing is a better determination of the proper motions. In Freire et al. (2001b), the number and precision of proper motions was small and only the motion of the GC as a whole was detectable. With a few more years of intense timing with the hAFB, some of the proper motions were measured precisely enough to detect relative motions, particularly for 47 Tuc D, E and J (Freire et al. 2003).

Because of the increased timing baselines, the proper motions presented in this work (depicted graphically in Fig. 5) are significantly more precise. Although the proper motions themselves are displayed in J2000 equatorial coordinates, the error ellipses are aligned according to ecliptic coordinates, where the measurement uncertainties are least correlated. In Fig. 5, we display the 17 pulsars for which both proper motion 1- $\sigma$ Monte Carlo uncertainties 
Table 3 - continued

\begin{tabular}{|c|c|c|c|}
\hline Pulsar & 47 Tuc $\mathrm{T}$ & 47 Tuc U & 47 Tuc Y \\
\hline Right Ascension, $\alpha(\mathrm{J} 2000)$ & $00: 24: 08.5491(5)$ & $00: 24: 09.8366(1)$ & $00: 24: 01.4026(1)$ \\
\hline 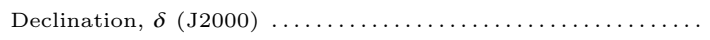 & $-72: 04: 38.932(3)$ & $-72: 03: 59.6882(4)$ & $-72: 04: 41.8363(4)$ \\
\hline Proper Motion in $\alpha, \mu_{\alpha}\left({\left.\operatorname{mas~} \mathrm{yr}^{-1}\right) \ldots \ldots \ldots \ldots \ldots \ldots \ldots \ldots \ldots \ldots \ldots \ldots}\right.$ & $5.1(6)$ & $4.6(2)$ & $4.4(1)$ \\
\hline Proper Motion in $\delta, \mu_{\delta}\left({\left.\operatorname{mas~} \mathrm{yr}^{-1}\right) \ldots \ldots \ldots \ldots \ldots \ldots \ldots \ldots \ldots \ldots \ldots \ldots \ldots}\right.$ & $-2.6(7)$ & $-3.8(1)$ & $-3.4(1)$ \\
\hline 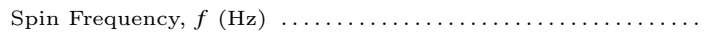 & $131.77869947406(2)$ & $230.264772211776(6)$ & $455.23717843241(1)$ \\
\hline First Spin Frequency derivative, $\dot{f}\left(10^{-15} \mathrm{~Hz} \mathrm{~s}^{-1}\right) \ldots \ldots \ldots \ldots$ & $-5.1021(2)$ & $-5.04916(9)$ & $7.2891(2)$ \\
\hline Second Spin Frequency derivative, $\ddot{f}\left(10^{-27} \mathrm{~Hz} \mathrm{~s}^{-2}\right) \ldots \ldots \ldots$ & $-3(2)$ & $18.8(6)$ & $-21.1(9)$ \\
\hline Start of Timing Data (MJD) $\ldots \ldots \ldots \ldots \ldots \ldots \ldots \ldots \ldots \ldots \ldots$ & 50683.712 & 48515.506 & 50739.663 \\
\hline End of Timing Data (MJD) $\ldots \ldots$ & 56466.934 & 56466.919 & 56508.973 \\
\hline Dispersion Measure, DM $\left(\mathrm{pc} \mathrm{cm}^{-3}\right)$ & $24.41(2)$ & $24.337(4)$ & $24.468(4)$ \\
\hline 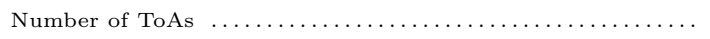 & 554 & 1309 & 804 \\
\hline 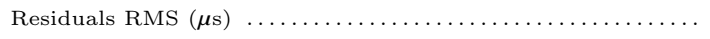 & 54.36 & 9.68 & 8.11 \\
\hline \multicolumn{4}{|c|}{ Binary Parameters } \\
\hline 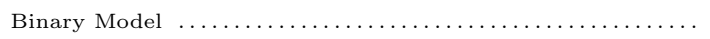 & ELL1 & ELL1 & ELL1 \\
\hline Projected Semi-major Axis, $x_{p}$ (lt-s) $\ldots \ldots \ldots \ldots \ldots$ & $1.338501(5)$ & $0.5269494(7)$ & $0.6685965(7)$ \\
\hline First Laplace-Lagrange parameter,$\eta \ldots \ldots \ldots \ldots \ldots \ldots$ & $3.55(7) \times 10^{-4}$ & $-2.9(4) \times 10^{-5}$ & $-3(3) \times 10^{-6}$ \\
\hline Second Laplace-Lagrange parameter,$\kappa \ldots \ldots \ldots \ldots \ldots \ldots$ & $1.85(7) \times 10^{-4}$ & $1.43(2) \times 10^{-4}$ & $0(2) \times 10^{-6}$ \\
\hline Epoch of passage at Ascending Node, $T_{\text {asc }}$ (MJD) & $51600.5692696(7)$ & $51600.3893516(1)$ & $51554.8340067(2)$ \\
\hline Rate of periastron advance, $\dot{\omega}(\mathrm{deg} / \mathrm{yr}) \ldots \ldots \ldots$ & - & $1.17(32)$ & - \\
\hline 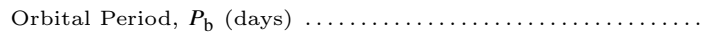 & $1.126176771(1)$ & $0.42910568324(8)$ & $0.5219386107(1)$ \\
\hline Orbital Period derivative, $\dot{P}_{b}\left(10^{-12} \mathrm{~s} \mathrm{~s}^{-1}\right) \ldots \ldots \ldots \ldots \ldots$ & $2.5(1.1)$ & $0.66(5)$ & $-0.82(7)$ \\
\hline \multicolumn{4}{|c|}{ Derived Parameters } \\
\hline Angular offset from centre in $\alpha, \theta_{\alpha}$ (arcmin) & +0.2215 & +0.3207 & -0.3283 \\
\hline Angular offset from centre in $\delta, \theta_{\delta}(\operatorname{arcmin}) \ldots \ldots \ldots \ldots$ & +0.2280 & +0.8821 & +0.1799 \\
\hline Total angular offset from centre, $\theta_{\perp}(\operatorname{arcmin}) \ldots \ldots \ldots \ldots$ & 0.3179 & 0.9386 & 0.3743 \\
\hline Total angular offset from centre, $\theta_{\perp}$ (core radii) $\ldots \ldots \ldots \ldots$ & 0.9160 & 2.7049 & 1.0788 \\
\hline Projected distance from centre, $r_{\perp}(\mathrm{pc}) \ldots \ldots \ldots \ldots \ldots \ldots \ldots$ & 0.4336 & 1.2805 & 0.5107 \\
\hline 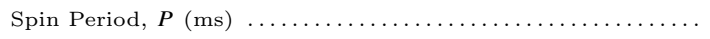 & $7.5884798073671(9)$ & $4.3428266963923(1)$ & $2.19665714352124(6)$ \\
\hline First Spin Period derivative, $\dot{P}\left(10^{-21} \mathrm{~s} \mathrm{~s}^{-1}\right) \ldots \ldots \ldots \ldots \ldots$ & $293.80(1)$ & $95.228(2)$ & $-35.1720(8)$ \\
\hline Line of sight acceleration from cluster field, $a_{\ell, \mathrm{GC}}\left(10^{-9} \mathrm{~m} \mathrm{~s}^{-2}\right)$ & $7.7(3.5)$ & $5.31(38)$ & $-5.4(4)$ \\
\hline Intrinsic spin period derivative, $\dot{P}_{\text {int }}\left(10^{-21} \mathrm{~s} \mathrm{~s}^{-1}\right) \ldots \ldots \ldots \ldots$ & $99(89)$ & $18(5)$ & $4.7(3.3)$ \\
\hline Characteristic Age, $\tau_{\mathrm{c}}(\mathrm{Gyr}) \ldots \ldots \ldots \ldots \ldots \ldots$ & $>0.43$ & 3.8 & $>3.1$ \\
\hline Line-of-sight jerk, $\dot{a}_{\ell}\left(10^{-21} \mathrm{~m} \mathrm{~s}^{-3}\right) \ldots \ldots \ldots \ldots \ldots \ldots \ldots \ldots \ldots \ldots \ldots \ldots \ldots$ & $7.8(3.7)$ & $-24.4(8)$ & $13.9(6)$ \\
\hline 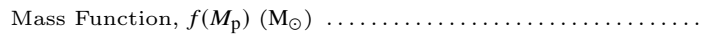 & $0.002030139(25)$ & $0.0008532200(35)$ & $0.0011779754(37)$ \\
\hline Minimum companion mass, $M_{\mathrm{c}, \min }\left(\mathrm{M}_{\odot}\right) \ldots \ldots \ldots \ldots \ldots$ & 0.171 & 0.126 & 0.141 \\
\hline Median companion mass, $M_{\mathrm{c}, \text { med }}\left(\mathrm{M}_{\odot}\right) \ldots \ldots \ldots \ldots \ldots \ldots$ & 0.200 & 0.146 & 0.164 \\
\hline 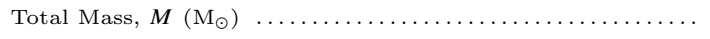 & - & $1.7(7)$ & - \\
\hline
\end{tabular}

(in ecliptic longitude, $\lambda$, and ecliptic latitude, $\beta$ ) are smaller

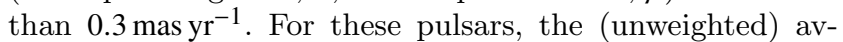
erage proper motion is $\mu_{\alpha}=5.00 \mathrm{mas} \mathrm{yr}^{-1}$ and $\mu_{\delta}=$

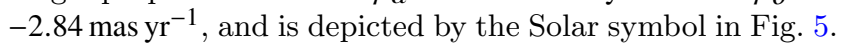
This represents the simplest estimate for the proper motion of the cluster as a whole, and it is consistent with the estimate presented in Freire et al. (2003): $\mu_{\alpha}=5.3 \pm 0.6$ mas yr $^{-1}$ and $\mu_{\delta}=-3.3 \pm 0.6 \mathrm{mas} \mathrm{yr}^{-1}$. The standard deviations of the proper motions around this average $\left(\sigma_{\mu}\right)$ are $0.59 \mathrm{mas} \mathrm{yr}^{-1}$ in $\alpha$ and $0.49 \mathrm{mas} \mathrm{yr}^{-1}$ in $\delta$. At a distance of $4.69 \mathrm{kpc}$ (Woodley et al. 2012), these standard deviations correspond to 13.2 and $10.9 \mathrm{~km} \mathrm{~s}^{-1}$ respectively. The uncertainty in the mean value is given by $\sigma_{\mu} / \sqrt{N}$, where $N$ is the number of measurements (17, in this case). Thus our uncertainties for the mean cluster motion are $\sigma_{\mu_{\alpha}}=0.14 \mathrm{mas} \mathrm{yr}^{-1}$ and $\sigma_{\mu_{\delta}}=0.12$ mas yr$^{-1}$.

An alternative method to estimate the overall motion of the cluster is to require that all observed pulsar proper mo- tions fit within the smallest possible velocity envelope. This corresponds to finding the centre of a circle defined by the proper motions of the three outermost pulsars in the proper motion plot, namely 47 Tuc D, E and U (alternatively we can use 47 Tuc D, N and U; we prefer the former set because the proper motion for 47 Tuc $\mathrm{E}$ is known much more precisely). This minimal proper motion envelope is represented by the dashed circle and has a radius of $1.10 \mathrm{mas} \mathrm{yr}^{-1}$; at $4.69 \mathrm{kpc}$ this represents a velocity of $24.5 \mathrm{~km} \mathrm{~s}^{-1}$, or about half of the escape velocity from the centre of the cluster $\left(\sim 50 \mathrm{~km} \mathrm{~s}^{-1}\right.$, e.g., McLaughlin et al. 2006). The centre of this minimal envelope is at $\mu_{\alpha}=5.16 \mathrm{mas} \mathrm{yr}^{-1}$ and $\mu_{\delta}=-2.85 \mathrm{mas} \mathrm{yr}^{-1}$, represented by a solid dot at the centre of Fig. 5. This $\mu_{\alpha}$ is almost $1-\sigma$ consistent with the average estimated above, the $\mu_{\delta}$ is practically identical to the average. 
Table 4. Timing parameters for the four black-widow systems with timing solutions in 47 Tuc, as obtained from fitting the observed ToAs with TEMPO (for the fifth black widow system, 47 Tuc P, it was possible to derive a precise orbit but no phase-coherent solution, see Paper I). For the characteristic age, we either present the median or a 2- $\sigma$ lower limit. The orbital models used are the ELL1 (Lange et al. 2001) and BTX (D. Nice, unpublished); for the latter the orbital periods are derived from the orbital frequency and presented in square brackets.

\begin{tabular}{|c|c|c|c|c|}
\hline Pulsar & 47 Tuc I & 47 Tuc $\mathrm{J}$ & 47 Tuc $\mathrm{O}$ & 47 Tuc $\mathrm{R}$ \\
\hline Right Ascension, $\alpha(\mathrm{J} 2000)$ & $00: 24: 07.9347(2)$ & $00: 23: 59.4077(1)$ & $00: 24: 04.65254(6)$ & $00: 24: 07.5851(2)$ \\
\hline Declination, $\delta(\mathrm{J} 2000) \ldots \ldots \ldots \ldots \ldots \ldots$ & $-72: 04: 39.6815(7)$ & $-72: 03: 58.7908(5)$ & $-72: 04: 53.7670(2)$ & $-72: 04: 50.3954(5)$ \\
\hline Proper Motion in $\alpha, \mu_{\alpha}\left(\operatorname{mas~} \mathrm{yr}^{-1}\right)$ & $5.0(2)$ & $5.27(6)$ & $5.01(5)$ & $4.8(1)$ \\
\hline Proper Motion in $\delta, \mu_{\delta}\left(\right.$ mas $\left.\mathrm{yr}^{-1}\right)$ & $-2.1(2)$ & $-3.59(9)$ & $-2.58(8)$ & $-3.3(2)$ \\
\hline 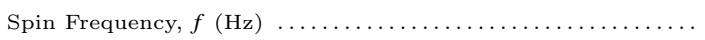 & $286.94469953049(1)$ & $476.04685844061(1)$ & $378.308788360098(6)$ & $287.31811946930(1)$ \\
\hline First Spin Frequency derivative, $\dot{f}\left(10^{-15} \mathrm{~Hz} \mathrm{~s}^{-1}\right) \ldots \ldots \ldots \ldots$ & $3.7771(2)$ & $2.2190(2)$ & $-4.34352(8)$ & $-12.2467(2)$ \\
\hline Second Spin Frequency derivative, $\ddot{f}\left(10^{-27} \mathrm{~Hz} \mathrm{~s}^{-2}\right) \ldots \ldots \ldots$ & $-33.5(9)$ & $20(1)$ & $43.8(5)$ & $-8.5(1.5)$ \\
\hline Start of Timing Data (MJD) $\ldots \ldots \ldots \ldots \ldots \ldots \ldots \ldots \ldots \ldots$ & 47859.462 & 47717.894 & 50683.712 & 50742.607 \\
\hline End of Timing Data (MJD) $\ldots \ldots \ldots \ldots \ldots \ldots \ldots \ldots \ldots \ldots \ldots \ldots$ & 56466.878 & 56388.106 & 56508.991 & 55362.896 \\
\hline Dispersion Measure, $\mathrm{DM}\left(\mathrm{pc} \mathrm{cm}^{-3}\right) \ldots \ldots \ldots \ldots \ldots \ldots \ldots \ldots \ldots \ldots \ldots \ldots \ldots$ & $24.43(1)$ & $24.588(3)$ & $24.356(2)$ & $24.361(7)$ \\
\hline Number of ToAs $\ldots \ldots \ldots \ldots \ldots \ldots$ & 1201 & 10135 & 1903 & 449 \\
\hline Residuals RMS $(\mu \mathrm{s}) \ldots \ldots \ldots \ldots \ldots$ & 18.26 & 4.89 & 9.70 & 10.81 \\
\hline \multicolumn{5}{|c|}{ Binary Parameters } \\
\hline 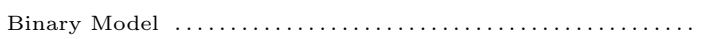 & ELL1 & BTX & BTX & ELL1 \\
\hline Projected Semi-major Axis, $x_{p}$ (lt-s) . & $3.8446(1) \times 10^{-2}$ & $4.04058(6) \times 10^{-2}$ & $4.51533(3) \times 10^{-2}$ & $3.3363(1) \times 10^{-2}$ \\
\hline 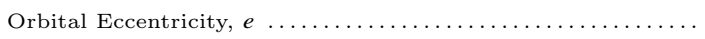 & - & 0 & 0 & - \\
\hline Longitude of Periastron, $\omega(\operatorname{deg}) \ldots \ldots \ldots \ldots \ldots \ldots \ldots \ldots$ & - & 0 & 0 & - \\
\hline Epoch of passage at Periastron, $T_{0}$ (MJD) $\ldots \ldots \ldots \ldots \ldots$ & - & $51600.1084250(6)$ & $51600.0757563(3)$ & - \\
\hline First Laplace-Lagrange parameter,$\eta \ldots \ldots \ldots \ldots \ldots \ldots$ & 0 & - & - & $-10(6) \times 10^{-5}$ \\
\hline Second Laplace-Lagrange parameter, $\kappa \ldots \ldots \ldots \ldots \ldots \ldots$ & 0 & - & - & $-3(7) \times 10^{-5}$ \\
\hline Epoch of passage at Ascending Node, $T_{\text {asc }}(\mathrm{MJD}) \ldots \ldots \ldots$ & $51600.002421(2)$ & - & - & $51600.0029871(6)$ \\
\hline Orbital Period, $P_{\mathrm{b}}$ (days) $\ldots \ldots \ldots \ldots \ldots \ldots \ldots \ldots$ & $0.2297922489(4)$ & {$[0.12066493766(13)]$} & {$[0.13597430589(9)]$} & $6.623147751(6) \times 10^{-2}$ \\
\hline Orbital Period derivative, $\dot{P}_{b}\left(10^{-12} \mathrm{~s} \mathrm{~s}^{-1}\right) \ldots$ & $-0.8(2)$ & - & - & $0.19(4)$ \\
\hline 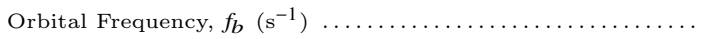 & - & $9.59191153(1) \times 10^{-5}$ & $8.511956725(6) \times 10^{-5}$ & - \\
\hline 1st Orbital Freq. derivative, $f_{b}^{(1)}\left(\mathrm{s}^{-2}\right) \ldots \ldots \ldots \ldots \ldots \ldots$ & - & $-21(34) \times 10^{-22}$ & $-7.3(1) \times 10^{-20}$ & - \\
\hline 2nd Orbital Freq. derivative, $f_{b}^{(2)}\left(\mathrm{s}^{-3}\right) \ldots \ldots \ldots \ldots \ldots \ldots \ldots \ldots$ & - & $-19(21) \times 10^{-29}$ & $-10(2) \times 10^{-29}$ & - \\
\hline 3rd Orbital Freq. derivative, $f_{b}^{(3)}\left(\mathrm{s}^{-4}\right) \ldots \ldots \ldots \ldots \ldots \ldots \ldots$ & - & $3.9(5) \times 10^{-35}$ & $33(15) \times 10^{-37}$ & - \\
\hline 4th Orbital Freq. derivative, $f_{b}^{(4)}\left(\mathrm{s}^{-5}\right) \ldots \ldots \ldots \ldots \ldots \ldots \ldots \ldots$ & - & $-15(24) \times 10^{-44}$ & - & - \\
\hline 5th Orbital Freq. derivative, $f_{b}^{(5)}\left(\mathrm{s}^{-6}\right) \ldots \ldots \ldots \ldots \ldots \ldots \ldots$ & - & $-5.5(8) \times 10^{-50}$ & - & - \\
\hline 6th Orbital Freq. derivative, $f_{b}^{(6)}\left(\mathrm{s}^{-7}\right) \ldots \ldots \ldots \ldots \ldots \ldots$ & - & $58(23) \times 10^{-59}$ & - & - \\
\hline 7 th Orbital Freq. derivative, $f_{b}^{(7)}\left(\mathrm{s}^{-8}\right) \ldots \ldots \ldots \ldots \ldots \ldots$ & - & $55(10) \times 10^{-66}$ & - & - \\
\hline 8th Orbital Freq. derivative, $f_{b}^{(8)}\left(\mathrm{s}^{-9}\right) \ldots \ldots \ldots \ldots \ldots \ldots \ldots$ & - & $-89(20) \times 10^{-74}$ & - & - \\
\hline 9th Orbital Freq. derivative, $f_{b}^{(9)}\left(\mathrm{s}^{-10}\right) \ldots \ldots \ldots \ldots \ldots \ldots$ & - & $-3.9(8) \times 10^{-80}$ & - & - \\
\hline 10th Orbital Freq. derivative, $f_{b}^{(10)}\left(\mathrm{s}^{-11}\right) \ldots \ldots \ldots \ldots \ldots \ldots$ & - & $86(15) \times 10^{-89}$ & - & - \\
\hline 11th Orbital Freq. derivative, $f_{b}^{(11)}\left(\mathrm{s}^{-12}\right) \ldots \ldots \ldots \ldots \ldots \ldots$ & - & $15(37) \times 10^{-96}$ & - & - \\
\hline 12th Orbital Freq. derivative, $f_{b}^{(12)}\left(\mathrm{s}^{-13}\right) \ldots \ldots \ldots \ldots \ldots \ldots$ & - & $-42(8) \times 10^{-104}$ & - & - \\
\hline \multicolumn{5}{|c|}{ Derived Parameters } \\
\hline Angular offset from centre in $\alpha, \theta_{\alpha}(\operatorname{arcmin}) \ldots \ldots \ldots \ldots$ & +0.1742 & -0.4821 & -0.0783 & +0.1473 \\
\hline Angular offset from centre in $\delta, \theta_{\delta}(\operatorname{arcmin}) \ldots \ldots \ldots \ldots$ & +0.2156 & +0.8972 & -0.0192 & +0.0371 \\
\hline Total angular offset from centre, $\theta_{\perp}(\operatorname{arcmin}) \ldots$ & 0.2772 & 1.0185 & 0.0806 & 0.1519 \\
\hline Total angular offset from centre, $\theta_{\perp}$ (core radii) & 0.7989 & 2.9352 & 0.2322 & 0.4378 \\
\hline Projected distance from centre, $r_{\perp}(\mathrm{pc}) \ldots \ldots \ldots \ldots \ldots \ldots \ldots$ & 0.3782 & 1.3895 & 0.1099 & 0.2072 \\
\hline 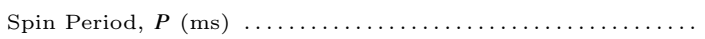 & $3.4849920616629(1)$ & $2.10063354535248(6)$ & $2.64334329724356(4)$ & $3.4804627074933(2)$ \\
\hline First Spin Period derivative, $\dot{P}\left(10^{-21} \mathrm{~s} \mathrm{~s}^{-1}\right) \ldots \ldots \ldots \ldots \ldots$ & $-45.873(2)$ & $-9.7919(9)$ & $30.3493(6)$ & $148.351(3)$ \\
\hline Line of sight acceleration from cluster field, $a_{\ell, \mathrm{GC}}\left(10^{-9} \mathrm{~m} \mathrm{~s}^{-2}\right)$ & $-11.8(3.7)$ & - & - & $10.1(1.9)$ \\
\hline Intrinsic spin period derivative, $\dot{P}_{\text {int }}\left(10^{-21} \mathrm{~s} \mathrm{~s}^{-1}\right) \ldots \ldots \ldots \ldots$ & $92(43)$ & - & - & $31(22)$ \\
\hline Characteristic Age, $\tau_{\mathrm{c}}(\mathrm{Gyr}) \ldots \ldots \ldots \ldots \ldots$ & 0.60 & - & - & $>0.73$ \\
\hline Line-of-sight jerk, $\dot{a}_{\ell}\left(10^{-21} \mathrm{~m} \mathrm{~s}^{-3}\right)$ & $35.0(1.0)$ & $-12.5(9)$ & $-34.68(37)$ & $8.9(1.5)$ \\
\hline Mass Function, $f\left(M_{\mathrm{p}}\right)\left(\mathrm{M}_{\odot}\right) \ldots \ldots \ldots$ & $0.0000011555(1)$ & $0.0000048646(2)$ & $0.0000053461(1)$ & $0.0000090898(10)$ \\
\hline Minimum companion mass, $M_{\mathrm{c}, \min }\left(\mathrm{M}_{\odot}\right) \ldots \ldots \ldots \ldots \ldots$ & 0.0132 & 0.0214 & 0.0221 & 0.0264 \\
\hline Median companion mass, $M_{\mathrm{c}, \text { med }}\left(\mathrm{M}_{\odot}\right) \ldots \ldots \ldots \ldots \ldots \ldots \ldots$ & 0.0153 & 0.0248 & 0.0256 & 0.0306 \\
\hline
\end{tabular}




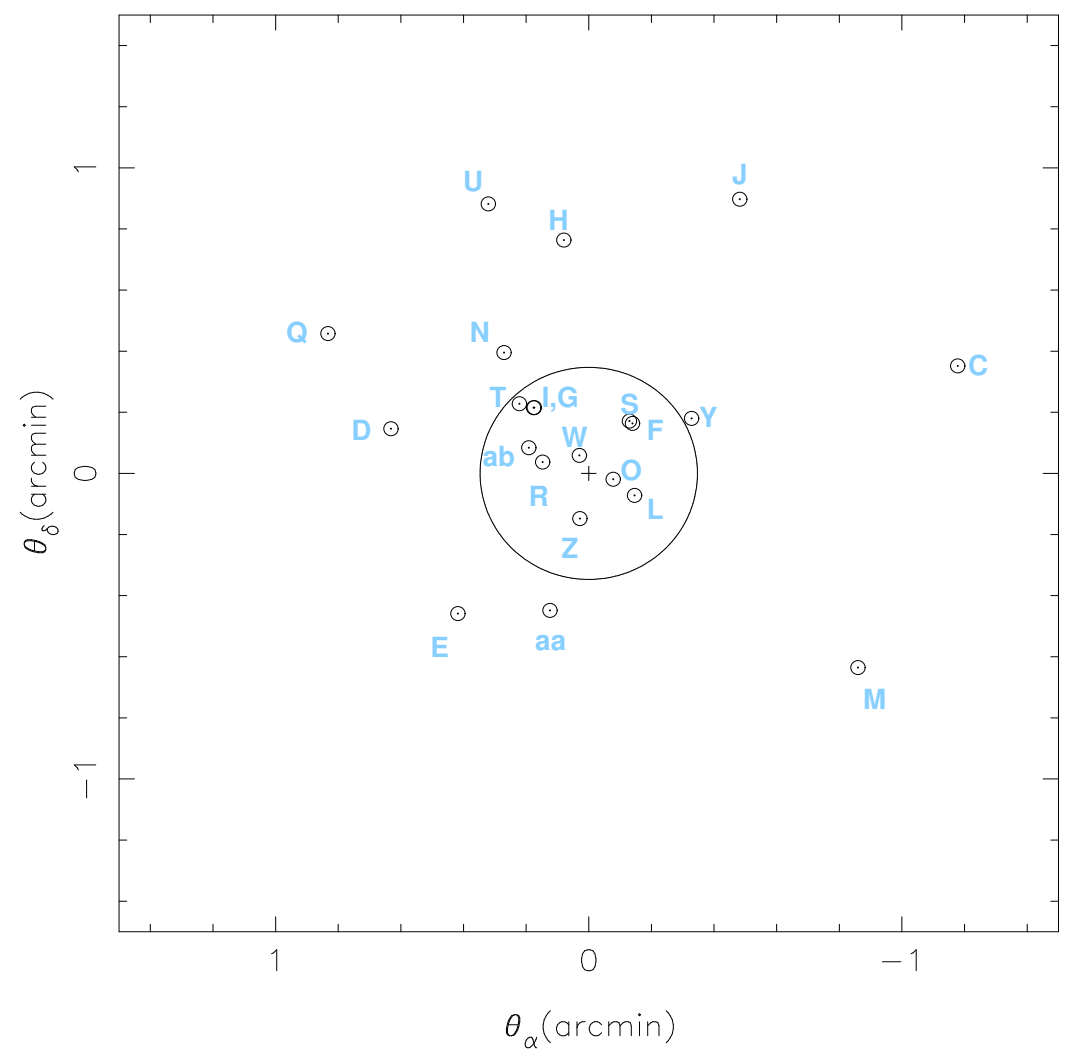

Figure 4. East-west $\left(\theta_{\alpha}\right)$ and north-south $\left(\theta_{\delta}\right)$ angular offsets from the centre of the GC 47 Tucanae for 22 of its 25 known pulsars. The central circle indicates the core radius. The $23^{\text {rd }}$ pulsar with a timing solution, 47 Tuc $\mathrm{X}$, is well outside the limits of this figure and its position relative to the cluster and the other pulsars is displayed graphically in Paper I (Ridolfi et al. 2016).

\subsection{Comparison with optical proper motions}

We now compare these numbers with previous literature. Regarding the absolute proper motion, the latest relevant study combines HIPPARCOS and GAIA positions to derive absolute proper motions for five Galactic globular clusters, among which is 47 Tuc (Watkins \& van der Marel 2016).

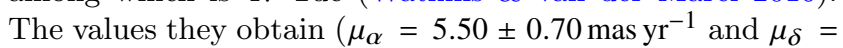
$-3.99 \pm 0.55$ mas yr$^{-1}$ ) are consistent with our measurement of the average proper motion in $\alpha$, but in $\delta$ the deviation is $-2.1 \sigma$, i.e., only marginally consistent. In Section 3.1 of that paper they list previous measurements of the proper motion of 47 Tuc and discuss their consistency, and it is clear that there is some disagreement between the proper motion estimates obtained by different methods. The situation will likely improve significantly with the second release of $G A I A$ data.

Our 1-D standard deviations for the proper motions agree with the $\sigma_{\mu, 0}$ obtained by Watkins et al. (2015a). This result agrees qualitatively with the observation by McLaughlin et al. (2006) that the observed velocity dispersion is largely constant across magnitude range, i.e., it appears to be the same for stellar populations of different masses.

\subsection{Proper motion pairs?}

Given the extreme proximity of 47 Tuc I and G in the sky and in acceleration, there is a suggestion that these pulsars could be in a bound system, with a semi-major axis $a_{\mathrm{p}}$ of at least 600 a.u. (Freire et al. 2001b). Such systems are not stable in 47 Tuc, since their cross section for violent interactions is too large, but they can exist temporarily. If this were the case, then the maximum relative orbital velocity should be of the order of $v \sim \sqrt{G M / a_{\mathrm{p}}}=2 \mathrm{~km} \mathrm{~s}^{-1}$. At the distance of 47 Tuc, this translates to an upper limit on the difference of proper motions of about $0.09 \mathrm{mas} \mathrm{yr}^{-1}$. As we can see from Fig. 5, the difference is of the order of $1 \mathrm{mas} \mathrm{yr}^{-1}$, ten times larger. We conclude therefore that, despite their proximity, these two pulsars are not in a bound system.

Two other pulsars, 47 Tuc F and S, are also remarkably close to each other and have identical DMs. In this case the minimum separation is 3700 a.u., requiring a maximum relative orbital velocity $v \sim 0.8 \mathrm{~km} \mathrm{~s}^{-1}$ and a maximal proper motion difference of $0.036 \mathrm{mas} \mathrm{yr}^{-1}$. Interestingly, this is not excluded by our measurements: as we can see in Fig. 5, the proper motion of 47 Tuc $\mathrm{F}$ falls within the 1- $\sigma$ contour for the proper motion of 47 Tuc S. The latter covers $1.1 \%$ of the proper motion surface within the velocity envelope determined above, so that is the probability of coincidence for any given pulsar. Given their spatial proximity, the proper motion coincidence is suggestive of a temporarily bound status.

As mentioned in Freire et al. (2001b), another test of the bound nature of these systems would be the detection of changes in their line-of-sight accelerations, which will produce a second derivative of the spin frequency, $\ddot{f}$. However, as we shall see in Section 4, the $\ddot{f}$ 's of these pulsars can be accounted for by their movement in the cluster potential. 


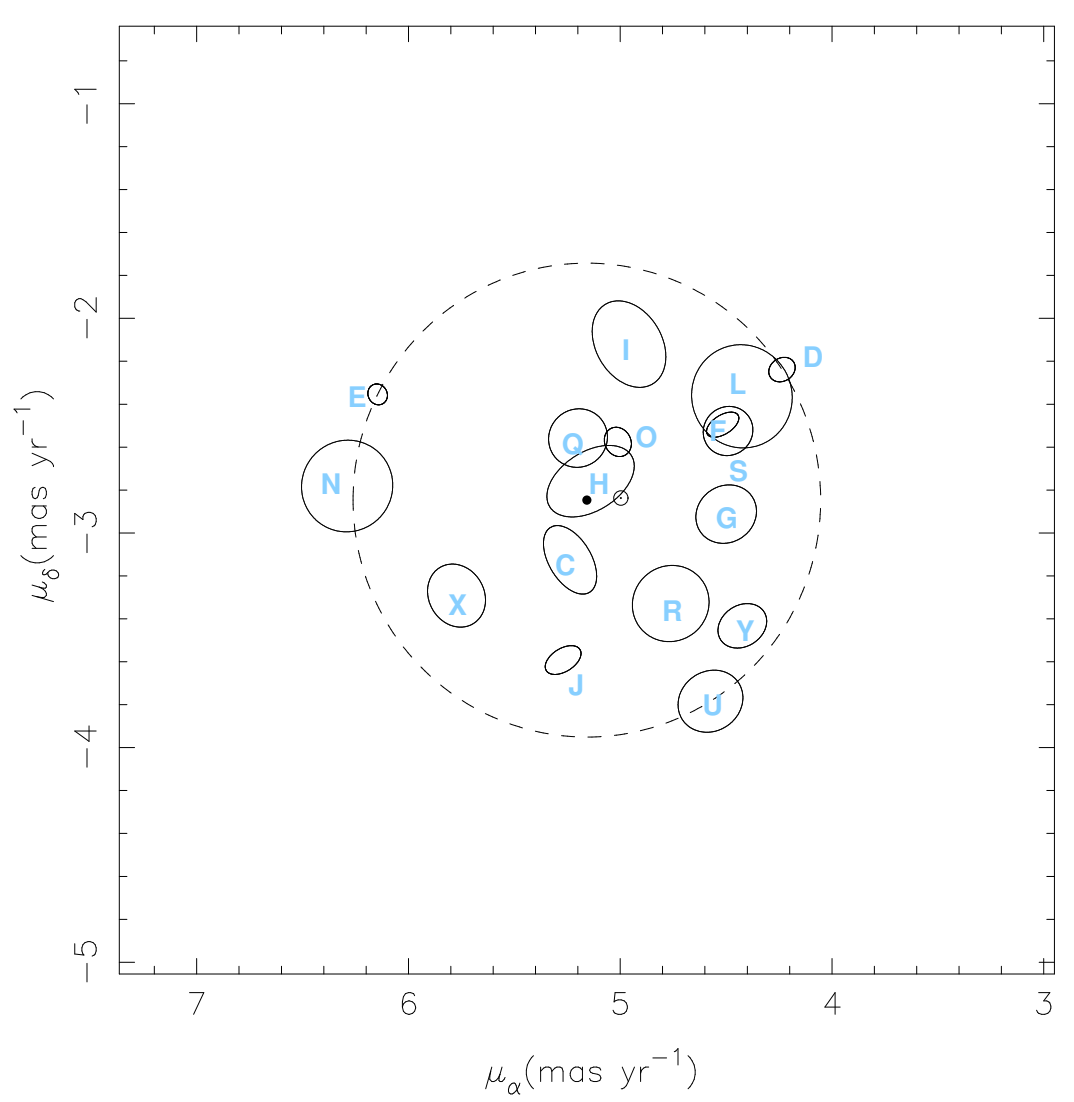

Figure 5. Proper motions for the 17 pulsars in 47 Tucanae where both $1-\sigma$ uncertainties are smaller than 0.3 mas yr ${ }^{-1}$. The proper motions are displayed by ellipses where the semi-axes have length of, and are aligned with, the 1- $\sigma$ proper motion uncertainties in ecliptic coordinates (where the positional and proper motion uncertainties are well defined). The differences in transverse velocity between the pulsars are highly significant, particularly for precisely timed pulsars like 47 Tuc D, E, F, J and O. The average of all proper motions in $\alpha$ and $\delta\left(\mu_{\alpha}=5.00 \mathrm{mas} \mathrm{yr}^{-1}\right.$ and $\left.\mu_{\delta}=-2.84 \mathrm{mas} \mathrm{yr}^{-1}\right)$ is given by the Solar symbol near the centre of the plot. This represents an estimate of the motion of the GC as a whole. The dashed circle represents the minimal possible velocity envelope for these pulsars, its centre (the solid dot at the centre of the plot, at $\mu_{\alpha}=5.16 \mathrm{mas} \mathrm{yr}^{-1}, \mu_{\delta}=-2.85 \mathrm{mas} \mathrm{yr}^{-1}$ ) represents another estimate of the proper motion of the globular cluster. This circle has a radius of $1.10 \mathrm{mas} \mathrm{yr}^{-1}$. At the assumed distance to 47 Tuc $(4.69 \mathrm{kpc})$ this is a velocity relative to the $\mathrm{GC}$ of $24.5 \mathrm{~km} \mathrm{~s}^{-1}$, or about half the escape velocity from the centre of the cluster.

\section{SPIN PERIOD DERIVATIVES}

We will now discuss the measurements of the spin frequency derivatives of the pulsars in 47 Tucanae. Like those of other pulsars in GCs (and unlike the spin period derivatives observed in the Galactic disk) the first spin frequency derivatives for the pulsars in 47 Tuc are mostly caused by dynamical effects. Higher spin frequency derivatives are, within our timing precision, caused entirely by dynamical effects.

\subsection{First spin period derivative and upper limits on acceleration in the cluster field}

The observed variation of spin period $\dot{P}_{\mathrm{obs}}$ is generally given by the following equation:

$\frac{\dot{P}_{\mathrm{obs}}}{P}=\frac{\dot{P}_{\mathrm{int}}}{P}+\frac{\mu^{2} d}{c}+\frac{a_{\ell, \mathrm{GC}}}{c}+\frac{a}{c}$,

where $P$ is the observed pulsar spin period, $\dot{P}_{\text {obs }}$ is the observed spin period derivative, $\dot{P}_{\text {int }}$ is the intrinsic spin period derivative, $\mu$ is the composite proper motion, $d$ is the distance to the cluster (the term $\mu^{2} d / c$ is known as the Shklovskii effect, see Shklovskii 1970), $c$ is the speed of light, $a_{\ell, \mathrm{GC}}$ is the line-of-sight acceleration of the pulsar in the gravitational field of the cluster and $a$ is the difference between the accelerations of the Solar System and 47 Tuc in the field of the Galaxy, projected along the direction to 47 Tuc ( $a=-1.172 \times 10^{-10} \mathrm{~m} \mathrm{~s}^{-2}$, calculated using the Reid et al. 2014 model for the Galactic rotation). In principle this equation could have other contributions, in particular accelerations caused by nearby stars. However, as shown by Phinney (1993), even in dense clusters those are very rarely relevant. As we shall see, the dominant term for the pulsars in 47 Tuc is $a_{\ell, \mathrm{GC}}$.

For most pulsars, we can only derive an upper limit on this dominant term from $\dot{P}_{\text {obs }} / P$, since $\dot{P}_{\text {int }}$ is generally not known but is always positive:

$a_{\ell, \max } \doteq a_{\ell, \mathrm{GC}}+\frac{\dot{P}_{\mathrm{int}}}{P} c=\frac{\dot{P}_{\mathrm{obs}}}{P} c-\mu^{2} d-a$,

these are displayed graphically in Fig. 6 as the triangles pointing down (to emphasise that they represent an upper limit on the cluster acceleration).

The solid lines represent the maximum line-of-sight acceleration due to the cluster potential $\left(a_{\ell}, \mathrm{GC}, \max \right)$ for the analytical model of the cluster described in Freire et al. (2005). 


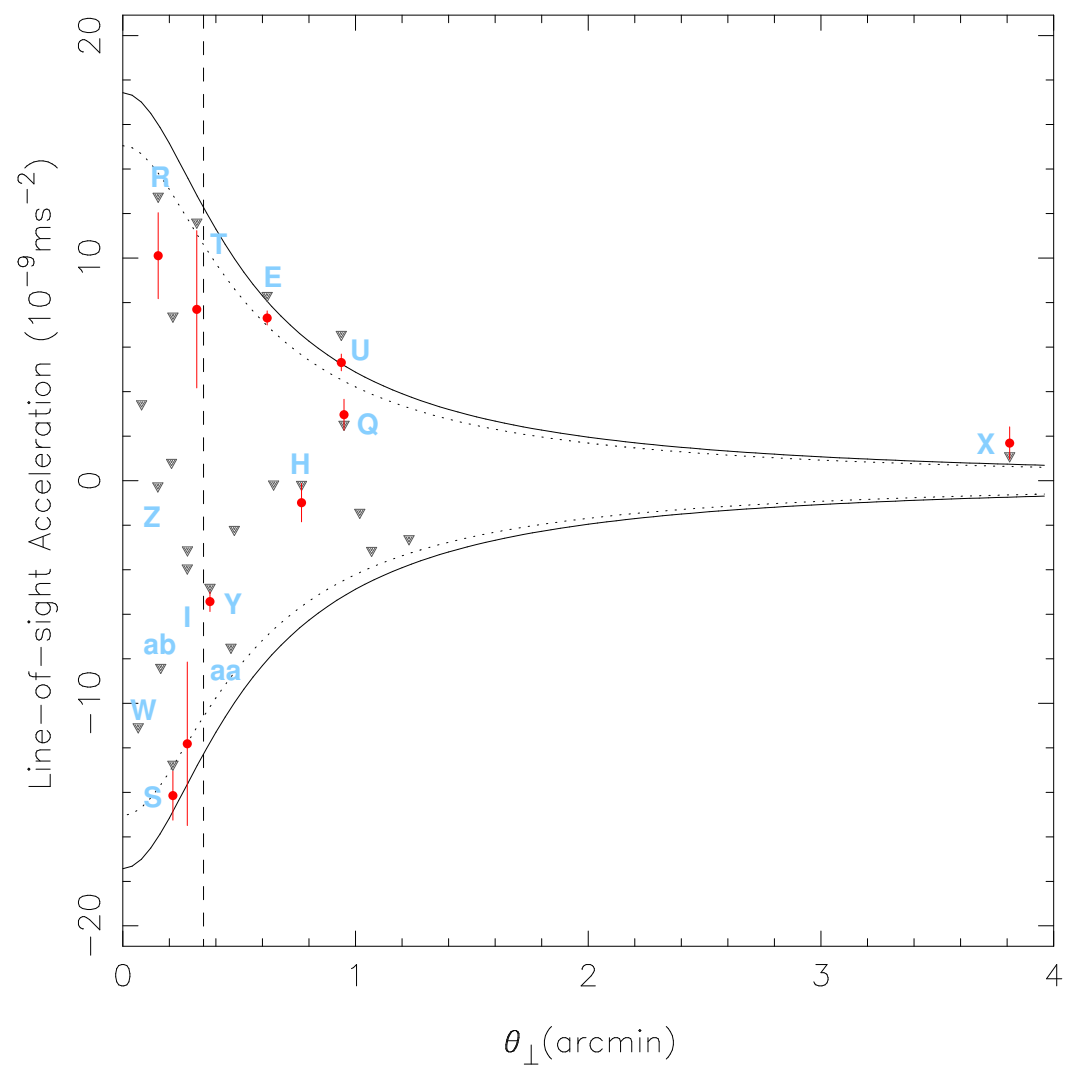

Figure 6. Line of sight accelerations $\left(a_{\ell}\right)$ as a function of the total angular offset from the centre of the cluster $\left(\theta_{\perp}\right)$ for the pulsars in 47 Tuc. The inverted triangles represent, for each pulsar system, an upper limit for its acceleration in the field of the cluster, this is determined from $\dot{P}_{\mathrm{obs}}$ (see discussion in Section 5). This is not a measurement of the real acceleration in the field of the cluster because of a contribution from the intrinsic spin period derivative of each pulsar $\left(\dot{P}_{\text {int }}\right)$. The red error bars represent measurements of the line-of-sight accelerations of 10 binary pulsars (47 Tuc E, H, I, Q, R, S, T, U, X and Y, which are named) determined from their orbital period derivatives, $\dot{P}_{\mathrm{b} \text {, obs }}$. We also plot the maximum and minimum accelerations $\left(a_{\ell}, \mathrm{GC}, \max \right)$ along each line of sight predicted by the analytical model of the cluster described in Section 4, with distances of $4.69 \mathrm{kpc}$ (solid lines) and $4.15 \mathrm{kpc}$ (dotted lines). We also name the systems with recently determined timing solutions $(\mathrm{R}, \mathrm{W}, \mathrm{Z}$, aa, ab). The core radius is indicated by the vertical dashed line.

This uses the mass distribution presented in King (1962) for the case where we are near (within $\sim 4$ core radii of) the centre of the cluster:

$\rho(x)=\frac{\rho(0)}{\left(1+x^{2}\right)^{3 / 2}}$,

where $x$ is the distance to the centre divided by the core radius $r_{c}=\theta_{c} d$. In this paper we use the $\rho(0)$ defined in eq. 1 . Since this is independent of distance, the mass of the cluster within a particular angular distance (i.e., within $x$ core radii, $\left.M_{\mathrm{GC}}(x)\right)$ is proportional to $d^{3}$, so the acceleration at that $x$ is proportional to $M_{\mathrm{GC}}(x) / d^{2}$, i.e., proportional to $d$ :

$a_{\mathrm{GC}}(x)=\frac{9 \sigma_{\mu, 0}^{2} d}{\theta_{c}} \frac{1}{x^{2}}\left(\frac{x}{\sqrt{1+x^{2}}}-\sinh ^{-1} x\right)$.

The line-of-sight component of this acceleration, $a_{\ell, \mathrm{GC}}(x)$, can be obtained by multiplying $a_{\mathrm{GC}}(x)$ by $\ell / x$, where $\ell$ is the distance (also in core radii) to the plane of the sky that passes through the centre of the cluster $(\Pi)$, such that $x=\sqrt{\ell^{2}+x_{\perp}^{2}}$ and $x_{\perp}=r_{\perp} / r_{c} \equiv \theta_{\perp} / \theta_{c}$. For each pulsar line-of-sight (characterized by a constant angular offset from the centre, $\left.\theta_{\perp}\right)$, we calculate $a_{\ell, \mathrm{GC}}(x)$ for a variety of values of $\ell$, recording the maximum and minimum values found, $a_{\ell, \mathrm{GC}, \max }$; these are the lines displayed in Fig. 6. For the line of sight going through the centre, we obtain the largest possible acceleration induced by the field of the cluster:

$a_{\ell, \mathrm{GC}, \max }(0)=1.5689 \frac{\sigma_{\mu, 0}^{2} d}{\theta_{c}} ;$

the numerical factor matches the more general expectation of $1.50 \pm 0.15$ from Eq. 3.6 in Phinney (1993). The latter was used to constrain the cluster parameters in Freire et al. (2003).

Apart from $d$, the predicted $a_{\ell, \mathrm{GC}, \max }\left(x_{\perp}\right)$ depend only on unambiguous angular measurements, this means that measurements of pulsar accelerations can be used to constrain $d$, i.e., this is a second kinematic distance measurement.

None of the pulsars, including those with recently published solutions (47 Tuc R, W, X, Y, Z, aa and ab, all named in Fig. 6) has a value of $a_{\ell \text {,max }}$ that is significantly larger than the model $a_{\ell, \mathrm{GC} \text {, max }}$ for its line of sight. The magnitude of the $a_{\ell, \mathrm{GC}}$ must be significantly larger than the contributions from $\dot{P}_{\text {int }}$, otherwise a majority of $\dot{P}_{\text {obs }}$ would be positive, while in fact similar numbers of pulsars have negative and positive $\dot{P}_{\text {obs }}$. For three pulsars, 47 Tuc E, U and X, the $a_{\ell, \max }$ is slightly larger than $a_{\ell, \mathrm{GC} \text { max }}$. For 47 Tuc E and U, 


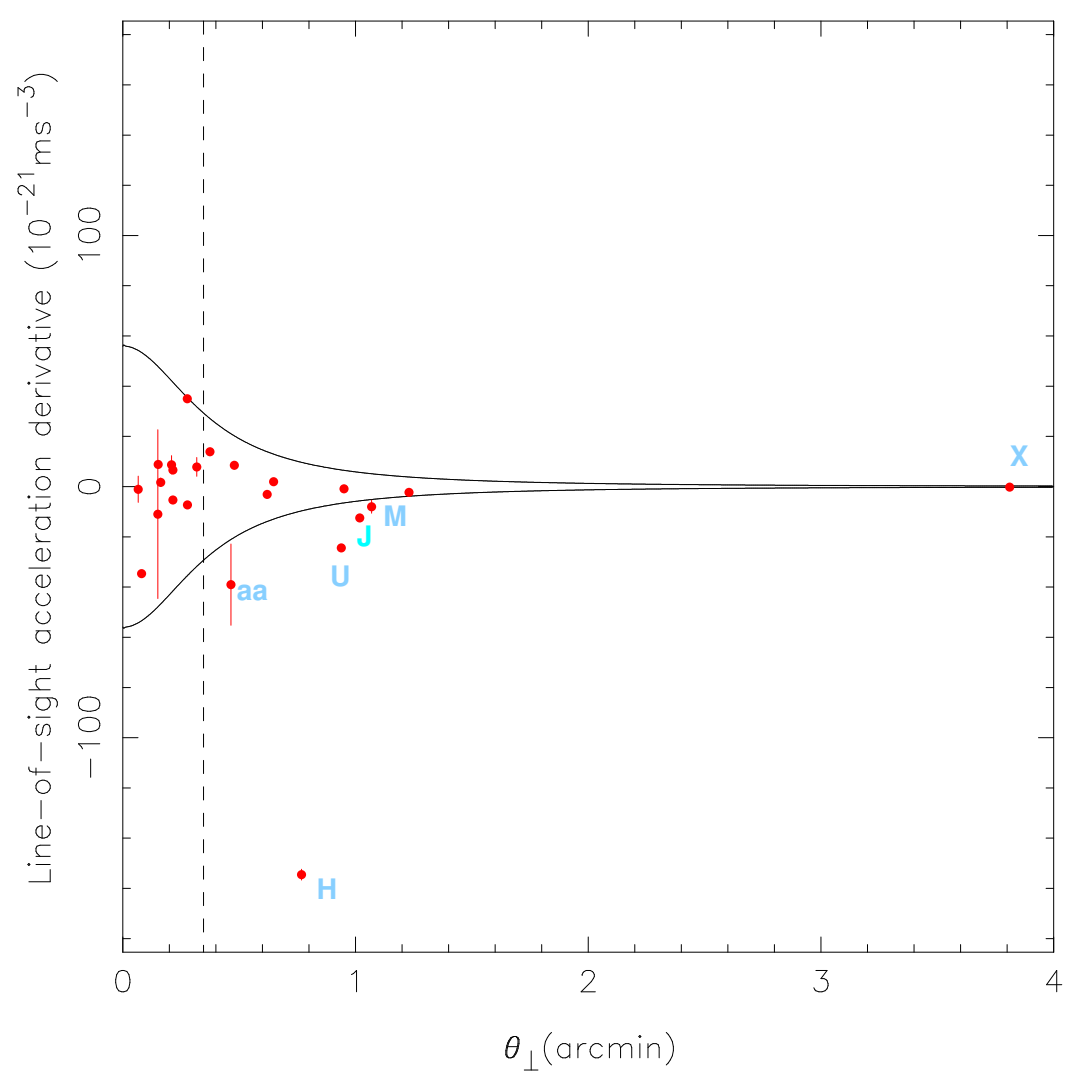

Figure 7. The red dots and error bars represent measurements and uncertainties of the line-of-sight jerks $\left(\dot{a}_{\ell}\right)$ for all the pulsars in 47 Tuc. The solid lines display, for each line of sight $\theta_{\perp}$, the maximum and minimum theoretical expectations (from Eq. 10) for the line-of-sight jerks caused by the motion of pulsars in the mean field of the cluster ( $\left.\dot{a}_{\ell}, \mathrm{GC}, \max \right)$. For some pulsars $(47 \mathrm{Tuc} \mathrm{H}$ and $\mathrm{U}$ and possibly 47 Tuc J), the observed $\dot{a}_{\ell}$ is larger than $\dot{a}_{\ell}$, GC, max ; this is likely due to the presence of stars near those systems. The core radius is indicated by the vertical dashed line.

this is caused by the contribution of their $\dot{P}_{\text {int }}$; as we will see in Section 5, their line-of-sight accelerations are (just about) consistent with the cluster model. For 47 Tuc X, it is likely that the same is happening, although in that case the $\dot{P}_{\mathrm{b} \text {, obs }}$ is not yet precise enough to reach any definite conclusions. However, it is unlikely that the analytical acceleration model described above is still entirely valid at its large $\theta_{\perp}$.

\subsection{Second spin frequency derivative and jerk}

For the vast majority of MSPs observed in the Galactic disk there is no detectable timing noise, even with timing precision much better than what we can achieve for the MSPs in 47 Tuc. This means that the large second spin frequency derivatives $(\ddot{f})$ observed for the latter are much more likely to reflect their rate of change of $a_{\ell}$, normally known as the (line-of-sight) "jerk" $\left(\dot{a}_{\ell}\right)$. Rearranging equation $(2)$ in (Joshi \& Rasio 1997), we get:

$\frac{\dot{a}_{\ell}}{c}=\left(\frac{\dot{f}}{f}\right)^{2}-\frac{\ddot{f}}{f} \simeq-\ddot{f} P$,

the approximation is valid since the first term, $(\dot{f} / f)^{2}$, is many orders of magnitude smaller than $\ddot{f} / f$. According to Phinney (1992), $\dot{a}_{\ell}$ has two main physical contributions. The first $\left(\dot{a}_{\ell, \mathrm{GC}}\right)$ arises from the movement of the pulsar in the potential of the cluster: different positions in the cluster will generally have a different $a_{\ell, \mathrm{GC}}$; the movement of the pulsar from one to the other will therefore cause a variation of this quantity. The second contribution to $\dot{a}_{\ell}$ is due to the gravity of nearby stars; this is more significant for denser clusters.

Freire et al. (2003) detected the second spin frequency derivative for only one pulsar, 47 Tuc $\mathrm{H}(\ddot{f}=1.6 \pm 0.2 \times$ $\left.10^{-25} \mathrm{~Hz} \mathrm{~s}^{-2}\right)$. They then estimated the maximum line-ofsight jerk induced by the motion of the pulsar in the mean field of the cluster $\left(\dot{a}_{\ell, \mathrm{GC}, \max }\right)$ and the corresponding $\ddot{f}$ $\left(\ddot{f}_{\text {max }}\right)$ using a slightly modified version of Equation 4.3 in Phinney (1993):

$\frac{\dot{a}_{\ell, \mathrm{GC}, \max }(0)}{c}=-\frac{\ddot{f}_{\max }}{f}=-\frac{4 \pi}{3} G \rho(0) \frac{v_{\ell, \max }}{c}$,

where $v_{\ell, \max }$ is the maximum velocity of a pulsar relative to the cluster, in this case assumed to be moving along the line of sight through the centre of the cluster. If $v_{\ell, \max }$ is positive (i.e., the pulsar is moving away from us), then $\dot{a}_{\ell, \mathrm{GC} \text {, max }}(0)$ is negative, and vice-versa.

Freire et al. (2003) used $\sigma_{0} \sim 13 \mathrm{~km} \mathrm{~s}^{-1}$ as an estimate of $v_{\ell, \max }$. The $\ddot{f}$ of 47 Tuc $\mathrm{H}$ is much larger than the resulting $\ddot{f}_{\text {max }}$, from this they concluded that this system is being perturbed by a nearby stellar companion.

However, that estimate of $\dot{a}_{\ell, \mathrm{GC}, \max }$ (and $\ddot{f}_{\max }$ ) is not very precise: First, because Eq. 8 applies only to the centre of the cluster; second because, as we have seen in Section 3, individual pulsars can have velocities along any direction that are almost twice as large as $\sigma_{0}$. Owing to our larger 
timing baseline $T$, we are now able to measure $\dot{a}_{\ell}$ precisely for almost all MSPs in 47 Tuc (see Tables 2 to 4); this improvement in measurements of $\dot{a}_{\ell}$ must be matched by an improvement in the prediction of $\dot{a}_{\ell}, \mathrm{GC}$, max .

This prediction is obtained from the gradient of $a_{\ell, \mathrm{GC}}$ along the direction of the line of sight $l \equiv \ell r_{c}$ where it reaches a maximum, at $l=\ell=0$ (i.e. in the plane $\Pi$, defined in section 4.1) and then multiplying it by $v_{\ell, \max }$. Near this plane $\ell$ is small, so $x=\sqrt{\ell^{2}+x_{\perp}^{2}} \simeq x_{\perp}$ is basically independent of $\ell$. In that case, the line-of-sight accelerations can be derived trivially from Eq. 5 multiplied by the projection factor $\ell / x_{\perp}$ :

$a_{\ell, \mathrm{GC}}\left(x_{\perp}\right)=\frac{9 \sigma_{\mu, 0}^{2} d}{\theta_{c}} \frac{\ell}{x_{\perp}^{3}}\left(\frac{x_{\perp}}{\sqrt{1+x_{\perp}^{2}}}-\sinh ^{-1} x_{\perp}\right)$,

Being proportional to $\ell$ in the vicinity of $\Pi$, these line-ofsight accelerations are zero for any object in $\Pi$, so the only non-zero spatial derivative of $a_{\ell, \mathrm{GC}}$ in that plane is along its normal: the direction of the line of sight $l$. This derivative is trivial since Eq. 9 is linear in $\ell$. Using $\mathrm{d} \ell / \mathrm{d} l=\mathrm{d} \ell /\left(d \theta_{c}\right) \mathrm{d} \ell=$ $1 /\left(d \theta_{c}\right)$, we obtain, for $\ell=0$ (not forgetting $v_{\ell \text {, max }}$ ):

$\dot{a}_{\ell, \mathrm{GC}, \max }\left(x_{\perp}\right)=\frac{9 \sigma_{\mu, 0}^{2}}{\theta_{c}^{2}} \frac{1}{x_{\perp}^{3}}\left(\frac{x_{\perp}}{\sqrt{1+x_{\perp}^{2}}}-\sinh ^{-1} x_{\perp}\right) v_{\ell, \max }$.

For the line of sight going through the centre $\left(x_{\perp}=\theta_{\perp}=0\right)$, Eq. 10 cannot be evaluated directly, but the limit of the terms with $x_{\perp}$ is $-1 / 3$. Thus, in that limit we recover the result of Eq. 8 (with the central density from Eq. 1) for the most extreme $\dot{a}_{\ell, \mathrm{GC}, \max }$ :

$\dot{a}_{\ell, \mathrm{GC}, \max }(0)=-\frac{3 \sigma_{\mu, 0}^{2}}{\theta_{c}^{2}} v_{\ell, \max }$.

Apart from $v_{\ell, \max }$ these predictions for $\dot{a}_{\ell, \mathrm{GC}, \max }$ depend only on angular measurements. In our calculations, we used $v_{\ell, \max }=v_{e}$, the velocity envelope derived in section 3 .

The comparison between this prediction and the measured jerks is displayed graphically in Fig. 7. The red dots and red vertical errorbars depict the measurement of the jerks and associated uncertainties. We also plot (in solid black lines) the $\dot{a}_{\ell, \mathrm{GC}}$, max predicted for each line of sight by our cluster model. Most pulsars have line-of-sight jerks that are smaller than our estimate of $\dot{a}_{\ell}, \mathrm{GC}$, max for their lines of sight; such jerks can therefore be attributed to the movement of the pulsars in the mean field of the cluster.

However, a few stand out. For 47 Tuc H, the observed $\ddot{f}$ is consistent with that reported in 2003 , but 10 times as precise: $\ddot{f}=1.60 \pm 0.02 \times 10^{-25} \mathrm{~Hz} \mathrm{~s}^{-2}$; the corresponding line-of-sight jerk $\left(\dot{a}_{\ell}=-1.545(22) \times 10^{-19} \mathrm{~m} \mathrm{~s}^{-3}\right)$ is much larger (in absolute terms) than $\left|\dot{a}_{\ell, \mathrm{GC} \text {, max }}\right|$ for that pulsar's line of sight (or any in the cluster), so we come to the same conclusion as Freire et al. (2003): this system must have a nearby companion. We can now see that this is also true for 47 Tuc U and J. For 47 Tuc M and aa the observed jerks are only $\sim 1 \sigma$ away from the $\dot{a}_{\ell, \mathrm{GC}, \max }$ for their lines of sight.

The systems with line-of-sight jerks larger than the maximum cluster mean-field expectation lie at distances from the core of about $1^{\prime}$, not near the centre. Given the larger density of stars near the centre one might expect that larger jerks would occur there, however the predicted meanfield jerks are also larger near the centre. The numerical simulations presented in Prager et al. (2016) suggest that the probability for a jerk from a nearby companion to be significantly larger than the cluster mean-field contribution is relatively flat with distance from the centre of the cluster. This means we are likely to find systems like 47 Tuc $\mathrm{H}$ at any distance from the centre.

\subsection{Third spin frequency derivative}

Unlike for the lower spin frequency derivatives, the third and higher spin frequency derivatives, $f^{(n)}$, can only be caused by the gravitational field of nearby objects.

The idea that 47 Tuc $\mathrm{H}$ is being influenced by a nearby stellar companion is supported by the fact that it is the pulsar in the cluster for which the measurement of $f^{(3)}$ is most significant, $f^{(3)}=(3.8 \pm 1.7) \times 10^{-35} \mathrm{~Hz} \mathrm{~s}^{-3}$, a $2.3-\sigma$ "detection". Most of the black widow systems also appear to have a non-zero $f^{(3)}$, but in no case is the measurement more significant than 2- $\sigma$. For the other main candidate for a stellar companion, 47 Tuc U, we measure $f^{(3)}=(-1.0 \pm$ 1.3) $\times 10^{-35} \mathrm{~Hz} \mathrm{~s}^{-3}$; we therefore did not fit for this parameter in the derivation of its timing solution (Table 3 ).

Since the uncertainty in the measurement of $f^{(3)}$ scales with $T^{-9 / 2}$, continued timing will improve these measurements very quickly. The rate of improvement will be even faster for higher frequency derivatives. The measurement of five such derivatives allows a unique determination of the five Keplerian orbital parameters (Joshi \& Rasio 1997); we would then know whether the 47 Tuc $\mathrm{H}$ system and the nearby star are bound or not.

\section{ORBITAL PERIOD DERIVATIVES}

One of the quantities that benefits most from prolonged timing is the measurement of the variation of the orbital period, $\dot{P}_{b}$. For most of the eclipsing binaries like 47 Tuc V and $\mathrm{W}$ (see Paper I) and 47 Tuc J and O (see Section 6), there are unpredictable variations in the orbital period with time, similar to those observed for other eclipsing binaries in the Galaxy (e.g., Shaifullah et al. 2016); in these cases we need many orbital frequency derivatives to describe the evolution of orbital phase with time. For the remaining binary pulsars - the MSP-WD systems (47 Tuc E, H, Q, S, T, U, X and Y) and two of the black widow systems (47 Tuc I and R) the phase evolution of the orbit can be described with a period and a period derivative only.

\subsection{Measurements of accelerations}

If the orbital period, $P_{\mathrm{b}}$, in the reference frame of the binary is stable, then we will not be able to measure orbital frequency derivatives higher than $1^{\text {st }}$ order (unless the system is in a triple - in which case the effects will be much more obvious in the spin frequency derivatives). At the Earth, the observed orbital period derivative will then be given by (Damour \& Taylor 1991):

$\frac{\dot{P}_{\mathrm{b}, \mathrm{obs}}}{P_{\mathrm{b}}}=\frac{\dot{P}_{\mathrm{b}, \mathrm{int}}}{P_{\mathrm{b}}}+\frac{\mu^{2} d}{c}+\frac{a_{\ell, \mathrm{GC}}}{c}+\frac{a}{c}$, 


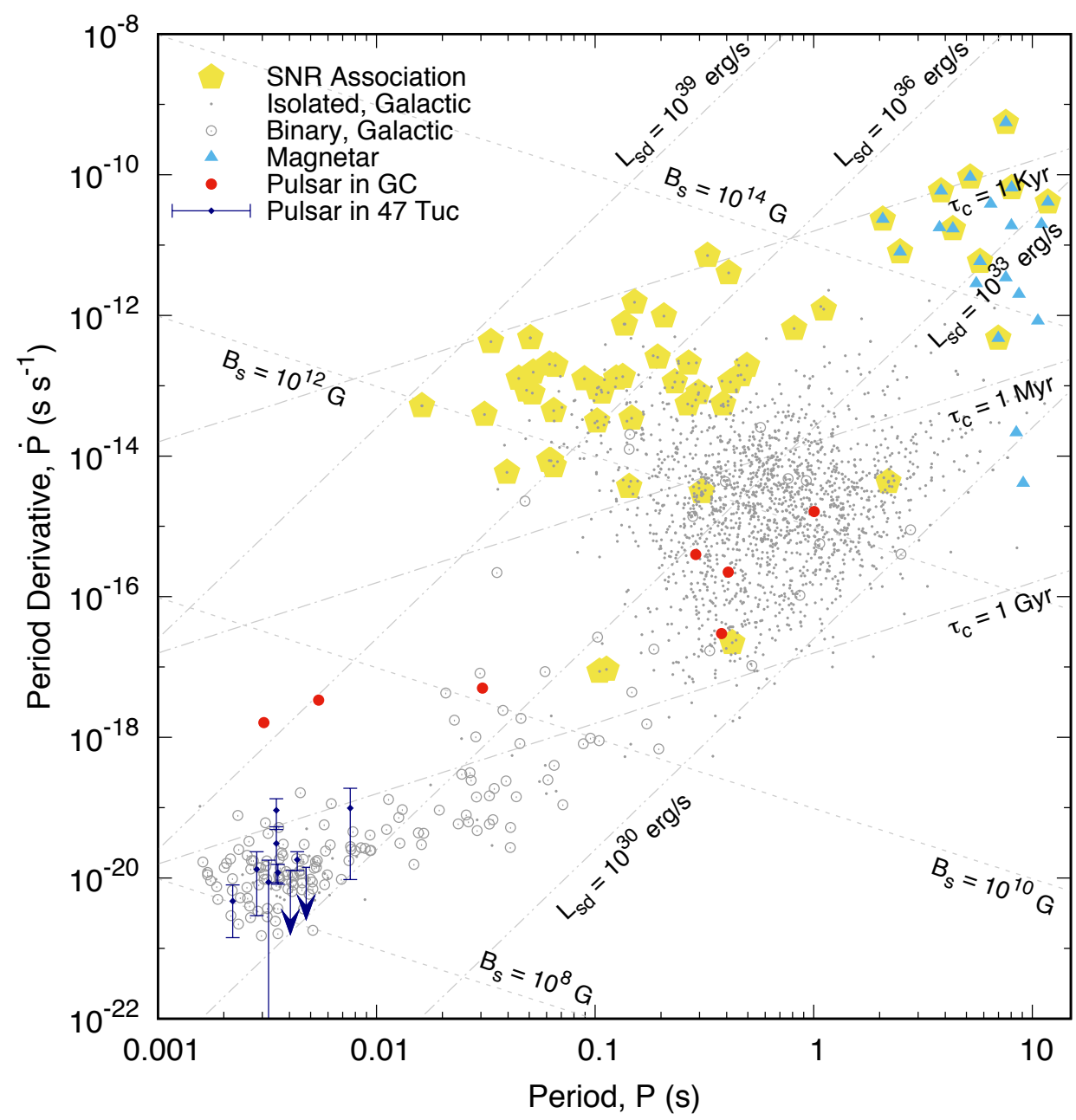

Figure 8. Period - period derivative plot for the pulsars in the ATNF Pulsar Catalogue (Manchester et al. 2005). The newly derived period derivatives for the MSPs in 47 Tuc (in dark blue) place them in the same region of the diagram where the majority of Galactic MSPs occur, i.e., they appear to be normal millisecond pulsars. Based on this sample, we conclude that 47 Tuc does not appear to have young pulsars like some seen in some other globular clusters (red dots).

where all parameters are as in equation (2), except that $\dot{P}_{\mathrm{b}, \mathrm{obs}}$ is the observed orbital period derivative and $\dot{P}_{\mathrm{b} \text { int }}$ is the intrinsic orbital period derivative. For the MSP-WD systems, the intrinsic variation of the orbital period, $\dot{P}_{\mathrm{b}, \text { int }}$, should be dominated by energy loss due to the emission of gravitational waves. This is expected to be a very small quantity: for the MSP-WD system with the shortest orbital period, 47 Tuc $\mathrm{U}\left(P_{\mathrm{b}}=0.42911 \mathrm{~d}\right)$, the orbital decay expected is $-1.36 \times 10^{-14} \mathrm{~s} \mathrm{~s}^{-1}$ (this assuming that the MSP has a mass of $1.4 M_{\odot}$ and an orbital inclination $i=90^{\circ}$ ), which is a factor of 2 smaller than the current measurement uncertainty for the $\dot{P}_{\mathrm{b} \text {,obs }}$ for that pulsar. The cases of 47 Tuc I and R are discussed in detail in Section 6.

Re-writing equation (12), and ignoring the intrinsic term, we can, for each pulsar, calculate the cluster acceleration, since the remaining terms are also known, in particular the proper motion (see Section 3):

$a_{\ell, \mathrm{GC}}=\frac{\dot{P}_{\mathrm{b}, \mathrm{obs}}}{P_{\mathrm{b}}} c-\mu^{2} d-a$.

These accelerations are presented in Tables 3 and 4, and depicted graphically as the vertical red error bars in Fig. 6 .
Like the values of $\dot{P}_{\text {obs }} / P$, they represent important constraints on the dynamics of the cluster. As we can see in Fig. 6, the accelerations for 47 Tuc S, E and U can (just about) be accounted for by the mass model for the cluster described in Section 4 with a distance of $4.69 \mathrm{kpc}$. With the kinematic distance $(4.15 \mathrm{kpc}$, represented by the dotted line in Fig. 6), this model cannot account for these accelerations.

We conclude therefore that our acceleration measurements are not compatible with a $d$ significantly smaller than $4.69 \mathrm{kpc}$, in agreement with most published distance estimates; they appear to be incompatible with the kinematic distance of $4.15 \mathrm{kpc}$. A more robust probabilistic estimate of the cluster distance most favoured by our measurements will be presented elsewhere.

\subsection{Intrinsic spin period derivatives}

As we can see from Fig. 6 , the measured values of $a_{\ell}$, GC tend to be similar, but slightly smaller than $a_{\ell \text {, max }}$. The difference, as can be seen in equation (3), is due to the contribution from $\dot{P}_{\text {int }}$. The values of $\dot{P}_{\text {int }}$ can be obtained directly from the observables by subtracting equation (12) from equation (2) 
and re-arranging the terms (and taking into account the fact that the $\dot{P}_{\mathrm{b} \text {,int }}$ are small):

$\dot{P}_{\text {int }}=\dot{P}_{\text {obs }}-\frac{\dot{P}_{\mathrm{b}, \text { obs }}}{P_{\mathrm{b}}} P$.

The intrinsic values of $\dot{P}$ are presented in Tables 3 and 4 . For most of the other pulsars, less constraining upper limits for $\dot{P}_{\text {int }}$ were derived assuming the largest possible negative value of $a_{\mathrm{GC}}$ for the line of sight of the pulsar (see Freire et al. 2001b and Freire et al. 2003).

Although the estimates of $\dot{P}_{\text {int }}$ are not measured with high significance (only a couple of cases, 47 Tuc E and U, are measured with 3- $\sigma$ significance), they already allow a comparison with the MSPs in the Galaxy. Putting the limits in a $P-\dot{P}$ diagram (Figure 8), we see that these pulsars have characteristics (spin-down energy, magnetic fields at the poles, characteristic ages) similar to the majority of MSPs in the disk of the Galaxy. They are very different from some of the "young" globular cluster pulsars (depicted in red), for which the $\dot{P}_{\text {obs }}$ are too large to be explained by cluster accelerations (for a discussion, see e.g., Freire et al. 2011, Johnson et al. 2013, Verbunt \& Freire 2014 and references therein).

The relatively large relative uncertainties of $\dot{P}_{\text {int }}$ imply that the derived magnetic fields, spin-down powers and characteristic ages of these pulsars still have large uncertainties. In what follows we calculate explicitly the characteristic ages, $\tau_{c}$, or lower limits on them, these are also presented in Tables 3 and 4 and were calculated using $\tau_{c}=P /\left(2 \dot{P}_{\text {int }}\right)$. The idea is to compare them with the total ages $\left(\tau_{o}\right)$ estimated for the WD companions that have been detected by the HST (Edmonds et al. 2001, 2002; Rivera-Sandoval et al. 2015; Cadelano et al. 2015). These estimates agree, i.e., we find no case where $\tau_{c}<<\tau_{o}$. A similar comparison was done in Rivera-Sandoval et al. (2015) and Cadelano et al. (2015) using preliminary numbers from our timing program. It is interesting to note that the two apparently oldest WD companions, 47 Tuc Q and Y, are those that have the largest lower limits for $\tau_{c}$.

\subsubsection{Tuc $Q$}

For 47 Tuc $\mathrm{Q}, \dot{P}_{\text {int }}=(-5.8 \pm 9.3) \times 10^{-21} \mathrm{~s} \mathrm{~s}^{-1}$. This means that we cannot specify an upper limit for $\tau_{c}$, since $\dot{P}_{\text {int }}$ could be very small. Its $2-\sigma$ upper limit, $1.28 \times 10^{-20} \mathrm{~s} \mathrm{~s}^{-1}$, implies a minimum $\tau_{c}$ of $5.0 \mathrm{Gyr}$.

For a variety of reasons, the $\tau_{o}$ for the WD companion of this pulsar is highly uncertain: the cooling age ranges from 0.3 to 5 Gyr (this value depends very sensitively on the mass of the WD), plus $\sim 1 \mathrm{Gyr}$ for the proto-WD phase (RiveraSandoval et al. 2015). We thus find that an age close to 6 Gyr is preferred for this system.

\subsubsection{Tuc $S$}

For 47 Tuc S, $\dot{P}_{\text {int }}=(1.3 \pm 1.0) \times 10^{-20} \mathrm{~s} \mathrm{~s}^{-1}$. Again, no reliable upper age can be derived, but a lower limit for $\tau_{c}$ of 1.3 Gyr can be derived from the $2-\sigma$ upper limit of $\dot{P}_{\text {int }}$. The cooling age ranges from 0.1 to $0.4 \mathrm{Gyr}$, to this we should add up to 0.4 Gyr for the time the companion spent as a proto-WD (Rivera-Sandoval et al. 2015). This suggests that $\tau_{o}$ is not larger than $0.8 \mathrm{Gyr}$. This is fine since $\tau_{c}$ assumes a starting spin period that is much shorter than the present spin period. This is clearly not the case for most MSPs, particularly those with shorter spin periods, meaning that the real age will generally be smaller than $\tau_{c}$.

\subsubsection{Tuc T}

For 47 Tuc $\mathrm{T}$, the timing constraints are not so precise and we get $\dot{P}_{\text {int }}=(9.9 \pm 8.9) \times 10^{-20} \mathrm{~s} \mathrm{~s}^{-1}$. This implies a $2-\sigma$ lower limit $\tau_{c}>0.43 \mathrm{Gyr}$. The estimated $\tau_{o}$ is from 0.1 to 0.8 Gyr (Rivera-Sandoval et al. 2015), consistent with $\tau_{c}$.

\subsubsection{Tuc $U$}

For 47 Tuc U, $\dot{P}_{\text {int }}=(1.82 \pm 0.55) \times 10^{-20} \mathrm{~s} \mathrm{~s}^{-1}$, this means we can establish solid lower and upper limits for $\tau_{c}$ from the $2-\sigma$ upper and lower limits of $\dot{P}_{\text {int }}: 2.4<\tau_{c}<9.4 \mathrm{Gyr}$. For the WD companion Rivera-Sandoval et al. (2015) derive a $\tau_{o}$ between $1.6-2.1 \mathrm{Gyr}$, slightly lower than $\tau_{c}$. As for 47 Tuc S, this is fine since $\tau_{c}$ represents an upper limit on the age that assumes a very small initial spin period.

\subsubsection{Tuс $Y$}

For this pulsar $\dot{P}_{\text {int }}=(4.7 \pm 3.3) \times 10^{-21} \mathrm{~s} \mathrm{~s}^{-1}$, from the $2-\sigma$ upper limit of $\dot{P}_{\text {int }}$ we derive a lower limit for $\tau_{c}$ of $3.1 \mathrm{Gyr}$. Rivera-Sandoval et al. (2015) derive a $\tau_{o}$ between 3.1 to 3.9 Gyr, in agreement with $\tau_{c}$.

\section{BLACK WIDOWS}

There are five black widow pulsars known in 47 Tuc, namely 47 Tuc I, J, O, and R (discussed below) and 47 Tuc P, studied in detail in Paper I. Black widow binary systems are mostly defined by their short orbital periods, small $\left(<0.05 M_{\odot}\right)$ companion masses, and the detectability of radio eclipses, although not for every system, while on the other hand redback pulsars have more massive companions $\left(>0.1 M_{\odot}\right)$ and always display eclipses (see e.g. Freire 2005; Roberts 2013 for reviews). The two redbacks in 47 Tuc, i.e. pulsars $\mathrm{V}$ and $\mathrm{W}$, were studied in detail in Paper I.

Both types of systems are known for their orbital variability: the orbital period (and sometimes the projected semi-major axis) change unpredictably with time, as seen in long-term timing of some black widow systems (see, e.g., Shaifullah et al. 2016). Such variability requires the use of the BTX orbital model (D. Nice, unpublished; http:// tempo.sourceforge.net/), which allows a description of the orbital behaviour using multiple orbital frequency derivatives. In order to characterise the orbital variability we also use a method described in Paper I (Section 5.2.1) and in Shaifullah et al. (2016), where we make multiple measurements of the time of ascending node, $T_{\text {asc }}$ as a function of time.

The results can be seen in Fig. 9, where we depict the orbital phase (and orbital period) evolution with time, and compare the observed $T_{\text {asc }}$ with the expectation based on the timing models in Table 4. The observed orbital phase variations are relatively smooth and are well described by the global models. 
47 Tuc I

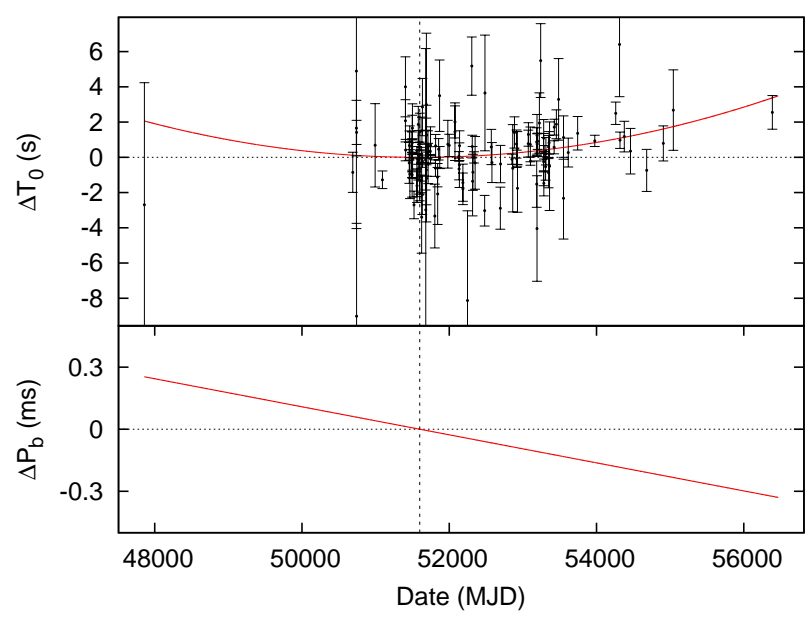

47 Tuc $\mathrm{O}$

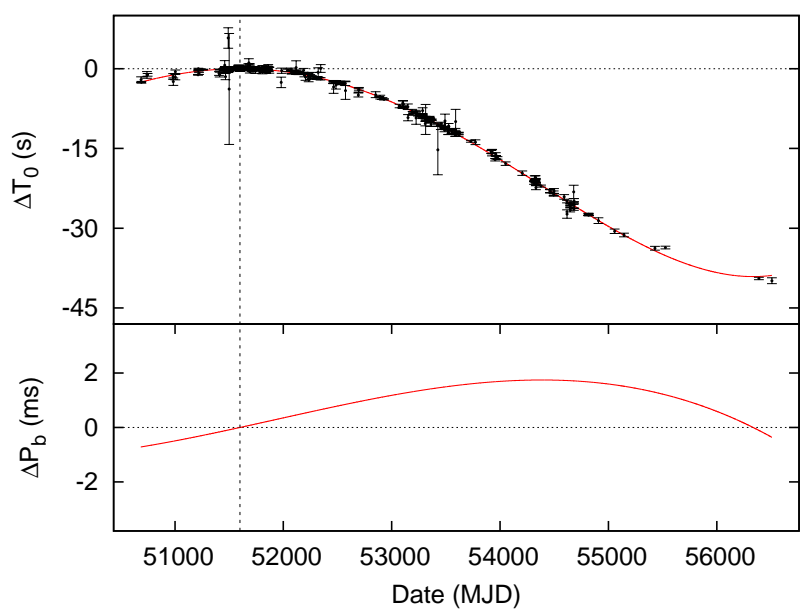

47 Tuc J

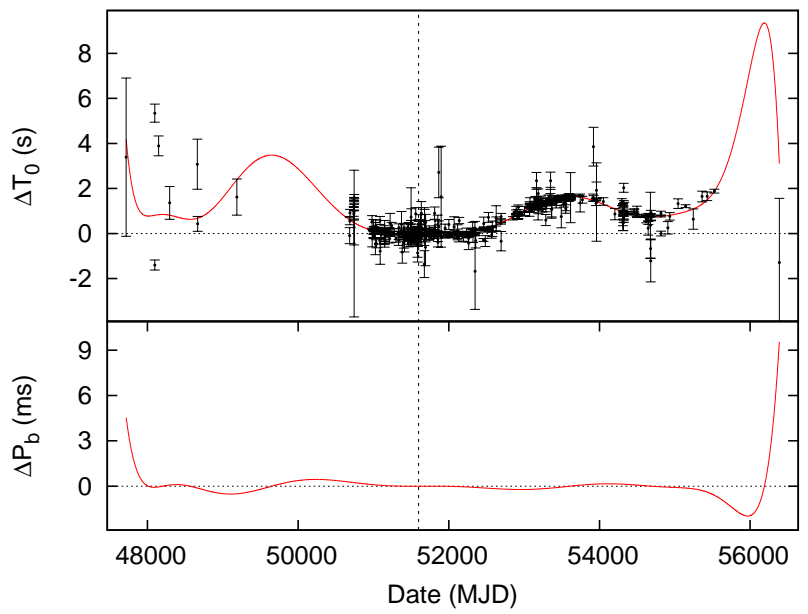

47 Tuc $\mathrm{R}$

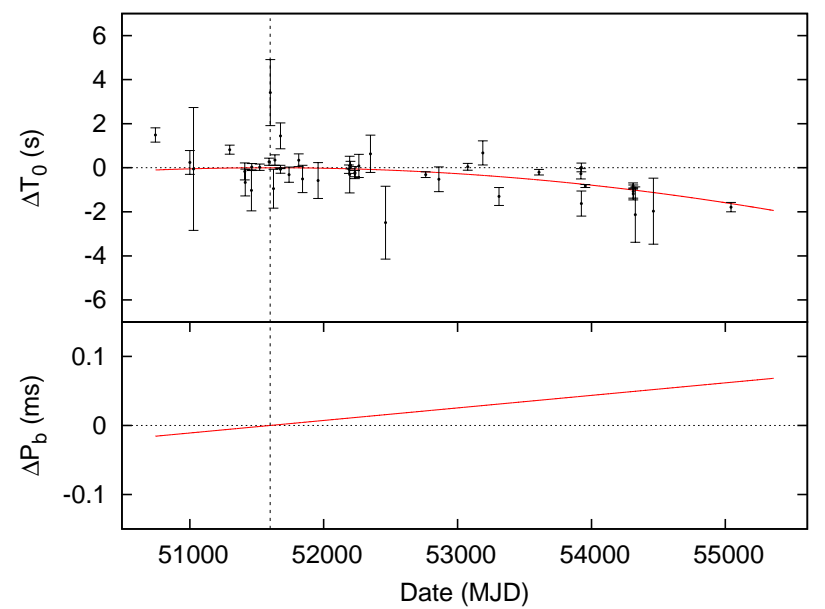

Figure 9. Orbital variability of the four black widows 47 Tuc I, J, O and R. In all the plots, the vertical dotted line indicates the passage through ascending node closest to the reference epoch, MJD $=51600$ (see Table 4). In the upper panels of each plot the vertical axis represents $\Delta T_{0}$ in the BTX model and $\Delta T_{\text {asc }}$ in the ELL1 model. For the lower panels of each plot the vertical axis represents the corresponding change in $P_{b}$. For the orbit closest to the reference epoch $\Delta T_{0} \equiv \Delta T_{\text {asc }}=0$ and $\Delta P_{\mathrm{b}}=0$. In the case of 47 Tuc J, given the large number of orbital frequency derivatives, the model predicts large orbital phase swings outside the timing baseline; this is not an accurate prediction of the system's orbital phase evolution.

\subsection{Black widows with large orbital variability}

As mentioned before, two of the black widow systems, 47 Tuc $\mathrm{J}$ and $\mathrm{O}$, display this characteristic variability. For 47 Tuc J, the orbital period appears to vary by a fraction of $1 \mathrm{~ms}$ in a quasi-sinusoidal fashion. Twelve orbital frequency derivatives were thus necessary to correctly model this behaviour within the timing baseline. The orbital period of 47 Tuc $\mathrm{O}$, instead, shows a constant increase until MJD 54300, then a decrease later. In this case, only three orbital frequency derivatives were necessary to model the variability. This is in part due to the fact that the timing baseline for this pulsar is shorter than for 47 Tuc J. These variations are not caused by any nearby objects, as the motion of the system would be obvious in variations of the observed pulse period.

It is important to note that, for these pulsars, the BTX models are only valid in the time span covered by the avail- able data, i.e. they do not have predictive power and cannot accurately describe the orbital phase evolution outside the timing baseline.

\subsection{Black widows with small orbital variability}

Even with the long timing baseline being considered in this paper, the orbits of 47 Tuc I and R can be described without the need of introducing any orbital frequency derivatives higher than the first (which in these cases we report as $\left.\dot{P}_{\mathrm{b}, \text { obs }}\right)$. This could be due, to some extent, to lack of timing precision. Looking at Fig. 9, we can see that the oscillations in $\Delta T_{\text {asc }}$ for 47 Tuc $\mathrm{J}$ are quite small compared to the dispersion of the data points observed in 47 Tuc I and R. If the latter could be timed with the same precision as 47 Tuc J, it is possible that subtle oscillations in $T_{\text {asc }}$ (such as those observed for 47 Tuc J) would become detectable.

However, the values of $\dot{P}_{\mathrm{b}, \text { obs }} / P_{\mathrm{b}}$ for these two systems 
are remarkably similar to their $\dot{P}_{\text {obs }} / P$ (see Fig. 6 ); they are even slightly smaller as one would expect from a positive intrinsic spin period derivative $\dot{P}_{\text {int }}$ : for 47 Tuc I, we obtain $\dot{P}_{\text {int }}=(9.2 \pm 4.3) \times 10^{-20} \mathrm{~s} \mathrm{~s}^{-1}$, and for 47 Tuc R $\dot{P}_{\text {int }}=$ $(3.1 \pm 2.2) \times 10^{-20} \mathrm{~s} \mathrm{~s}^{-1}$, values that are similar to those of the remaining pulsars. This makes it likely that, as in the case of $\dot{P}_{\mathrm{obs}} / P$, the $\dot{P}_{\mathrm{b} \text {,obs }} / P_{\mathrm{b}}$ observed in these two systems is mostly caused by the acceleration of the pulsars in the field of the globular cluster, $a_{\ell, \mathrm{GC}}$.

These systems have such short orbital periods that, despite the small companion masses, we must take into account an intrinsic variation of the orbital period caused by the emission of gravitational waves. In this case, equation (14) becomes:

$\dot{P}_{\text {int }}=\dot{P}_{\text {obs }}-\frac{\dot{P}_{\mathrm{b}, \text { obs }}-\dot{P}_{\mathrm{b}, \text { int }}}{P_{\mathrm{b}}} P$.

Assuming a pulsar mass of $1.4 M_{\odot}$ and an orbital inclination of $60^{\circ}$ for both pulsars and using the equations in Damour \& Taylor (1991), we obtain for the orbital decay $\dot{P}_{\mathrm{b} \text {,int }}=-4.8 \times 10^{-15} \mathrm{~s} \mathrm{~s}^{-1}$ for 47 Tuc I and $\dot{P}_{\mathrm{b}, \text { int }}=$ $-7.6 \times 10^{-14} \mathrm{~s} \mathrm{~s}^{-1}$ for 47 Tuc R. Inserting these terms in equation 15 , we obtain even smaller intrinsic spin period derivatives: $\dot{P}_{\text {int }}=(7.8 \pm 4.3) \times 10^{-20} \mathrm{~s} \mathrm{~s}^{-1}$ for 47 Tuc I and $\dot{P}_{\text {int }}=(-1.6 \pm 2.2) \times 10^{-20} \mathrm{~s} \mathrm{~s}^{-1}$ for 47 Tuc R, implying lower limits on the characteristic ages of both systems of 0.33 and 2.0 Gyr respectively. This means that the agreement between the cluster acceleration and the observed orbital period derivative is even more precise when we take the gravitational wave emission into account. We conclude, provisionally, that the black widow systems come in two flavours, with and without random orbital variability.

\section{NEW DETECTIONS OF THE RATE OF ADVANCE OF PERIASTRON}

Another measurement that benefits greatly from a much extended timing baseline is the rate of advance of periastron, $\dot{\omega}$. For a binary system consisting of two point masses (a reasonable approximation for the MSP-WD binaries in 47 Tuc), this is solely an effect of relativistic gravity. In general relativity, and to leading post-Newtonian order, it depends only on the Keplerian parameters and the total mass of the system $M$, in solar masses (Robertson 1938; Taylor \& Weisberg 1982):

$\dot{\omega}_{\mathrm{GR}}=3 \frac{\left(\mathrm{T}_{\odot} M\right)^{2 / 3}}{1-e^{2}}\left(\frac{P_{\mathrm{b}}}{2 \pi}\right)^{-5 / 3}$,

where $\mathrm{T}_{\odot} \equiv G M_{\odot} c^{-3}=4.9254909476412675 \mu$ s is a solar mass $\left(M_{\odot}\right)$ in time units, $c$ is the speed of light and $G$ is Newton's gravitational constant.

To measure this effect, we need a system with a significant orbital eccentricity $(e)$, otherwise it is impossible to measure the longitude of periastron $(\omega)$ with sufficient precision to detect its variation with time. For most MSPWD systems in the Galaxy, $e$ is too small for such a measurement to be feasible. In globular clusters, on the other hand, the stellar density is so high that binary pulsars are perturbed by close encounters with other members of the cluster; this will generally increase their orbital eccentricity (Phinney 1992; Phinney 1993). The large eccentricities of many binaries in GCs has allowed the measurement of their $\dot{\omega}$ - and consequently, of the binary masses (see e.g., Özel \& Freire 2016 and references therein). However, the perturbations (and corresponding increases in $e$ ) are larger for the wider binaries; this implies that, generally, when we are able to measure $\dot{\omega}$ well, then the wide orbit makes it hard to measure other relativistic parameters (these would be useful for determining the individual masses of the components of the binary). There are only two exceptions to date, both products of exchange interactions located in core-collapsed clusters (PSR J1807-2500B in NGC 6544, Lynch et al. 2012, and PSR B2127+11C in M15, Jacoby et al. 2006).

Among the known binary pulsars in 47 Tuc, the most eccentric by far is 47 Tuc $\mathrm{H}(e=0.0705585 \pm 0.0000007)$, which is also the second widest known in the cluster $\left(P_{\mathrm{b}}=\right.$ $2.3577 \mathrm{~d}$ ). This orbital eccentricity is $4-5$ orders of magnitude larger than observed in MSP-WD systems of similar $P_{\mathrm{b}}$ in the Galactic disk. For this system, Freire et al. (2003) measured $\dot{\omega}=0.0658 \pm 0.0009^{\circ} \mathrm{yr}^{-1}$ (where the uncertainty is the $1-\sigma$ error returned by TEMPO). This allowed an estimate of the total mass of the system of $M=1.61 \pm 0.03 M_{\odot}(1-\sigma)$. Our current value is a factor of five better: $\dot{\omega}=0.06725 \pm 0.00019^{\circ} \mathrm{yr}^{-1}$; this implies $M=1.665 \pm 0.007 M_{\odot}(1-\sigma)$. No other relativistic orbital effects are detectable, so it is not possible to determine the individual masses in this binary. However, combining this constraint with the constraint $\sin i \leq 1$, we obtain $M_{\mathrm{p}}<1.49 M_{\odot}$ and $M_{\mathrm{c}}>0.175 M_{\odot}$, for the mass of the pulsar and of the companion, respectively.

Although much lower, the eccentricities of most of the MSP-WD systems in 47 Tuc are also orders of magnitude larger than observed among MSP-WD systems with similar orbital periods in the Galactic disk. Because of this, we have made significant $(>3 \sigma)$ detections of $\dot{\omega}$ in 3 other systems: 47 Tuc $\mathrm{E}\left(\dot{\omega}=0.090 \pm 0.016^{\circ} \mathrm{yr}^{-1}, M=2.3 \pm 0.7 M_{\odot}\right)$, 47 Tuc $\mathrm{S}\left(\dot{\omega}=0.311 \pm 0.075^{\circ} \mathrm{yr}^{-1}, M=3.1 \pm 1.1 M_{\odot}\right)$ and 47 Tuc $\mathrm{U}\left(\dot{\omega}=1.17 \pm 0.32^{\circ} \mathrm{yr}^{-1}, M=1.7 \pm 0.7 M_{\odot}\right)$. These measurements are, however, not yet precise enough to derive any astrophysically interesting values of the total masses for these systems. Improving them is important, because if we can determine precise total masses for these systems, then we will have good estimates for the masses of these pulsars since their WD companion masses are relatively well known from optical photometry (Edmonds et al. 2002; Rivera-Sandoval et al. 2015; Cadelano et al. 2015).

Another two systems where $\dot{\omega}$ might be detectable in the future are 47 Tuc $\mathrm{Q}\left(0.46 \pm 0.22^{\circ} \mathrm{yr}^{-1}\right)$ and 47 Tuc T $(0.30 \pm$ $\left.0.28^{\circ} \mathrm{yr}^{-1}\right)$, again two systems for which we have good optical detections of the WD companions. For the remaining MSPWD systems (47 Tuc X and Y), the orbital eccentricities are too low for a measurement in the foreseeable future.

\section{DISCUSSION}

\subsection{What do the pulsars tell us about the cluster?}

The globular cluster 47 Tuc has one of the largest total stellar interaction rates $(\Gamma)$ among clusters in the Milky Way system (Verbunt \& Hut 1987; Bahramian et al. 2013). A consequence of this is that, following the many exchange encounters, many old, "dead" neutron stars find themselves 
in binaries with main sequence (MS) companions. Subsequent evolution of these companions causes transfer of gas to the NSs, i.e., the system becomes a low-mass X-ray binary (LMXB). After this, the companion typically becomes a low-mass WD, and the NS becomes a radio MSP. The large number of MSPs in 47 Tuc can therefore be understood primarily as a consequence of the large $\Gamma$.

The characteristic ages of the MSPs in 47 Tuc and the optical ages of their WD companions suggest that these systems have been forming at a near-constant rate throughout the age of the cluster, i.e., there is no indication of an early burst of MSP formation (which would make all pulsars look very old). There are also no signs of an ongoing burst of MSP formation either - none of the pulsars in the cluster has a large $\dot{P}_{\text {int }}$ that cannot be accounted for by the cluster acceleration model, none have characteristic ages smaller than about 0.33 Gyr (the lower limit for the age of 47 Tuc I). In this respect, the situation in 47 Tuc offers a stark contrast to that observed in some of the core-collapsed clusters, in particular NGC 6624, where at least three pulsars (out of the six known in that cluster) have characteristic ages smaller than 0.2 Gyr (Lynch et al. 2012), and in a particular case (PSR B1820-30A) as small as 25 Myr (Freire et al. 2011).

The difference between the populations of these clusters reflects fundamentally different dynamics. Although both types of clusters have a similar $\Gamma$, the interaction rate per binary, $\gamma$ (Verbunt \& Freire 2014) is much higher in NGC 6624 than in 47 Tuc. The fundamental reason for this is the fact that NGC 6624 has a collapsed core.

As already discussed in Paper I, the pulsar population in 47 Tuc has the characteristics one would expect for a GC with a low $\gamma$ : any newly formed LMXBs evolve undisturbed to their normal outcomes (MSP-WD binaries, black widows and isolated MSPs, as observed in the Galactic disk). All systems have large $\tau_{c}$ the moment they form. There are no mildly recycled pulsars - there are currently no companion stars in GCs massive enough (and evolving fast enough) to result in mild recycling, as seen for instance in double neutron star systems and pulsars with massive WD companions in the Galactic disk. This is the likely reason for the remarkably small range of spin periods $(1.8<P<7.6 \mathrm{~ms})$ for the pulsars in 47 Tucanae. Furthermore, the binary systems in 47 Tuc have relatively small orbital eccentricities compared to what we see in denser clusters, like Terzan 5 and M28 (for even denser clusters, binary destruction sets in, but we do see a few very eccentric survivors). The only "eccentric" binary in 47 Tuc, 47 Tuc H, might have gained its eccentricity from an object orbiting it, not from interactions with other stars.

In clusters with higher $\gamma$, we can find a higher percentage of isolated pulsars (from the disruption of MSP-WD systems), mildly recycled - and apparently young - pulsars (from the disruption of X-ray binaries, which leaves the recycling process incomplete) and products of secondary exchange interactions, i.e., exchange interactions that happen after the formation of the MSP. As discussed in Paper I, none of the MSPs in 47 Tuc is clearly the product of such an interaction.

Furthermore, in high- $\gamma$ GCs we find many pulsars very far from the cluster core. An extreme example is NGC 6752 (D'Amico et al. 2002; Corongiu et al. 2006), a core-collapsed cluster where two of the five known pulsars lie at more than
14 core radii from the centre. This phenomenon is common in other core-collapsed GCs and is caused by chaotic binary interactions, which typically have a strong recoil that can propel MSPs to the outer reaches of the cluster. In 47 Tuc, all pulsars but one appear to lie close to the core, their radial distribution being as expected from mass segregation of a dynamically relaxed population (Heinke et al. 2005). Even for the exceptional system, 47 Tuc X, it is not clear whether there was a recoil in the past (Paper I).

A detailed characterization of the pulsar populations of other GCs will be important for testing this general picture. The pulsar populations in low- $\gamma$ GCs (like M3, M5, M13, M22, M53, M62, NGC 6749 and NGC 6760) should have characteristics similar to 47 Tuc, thus different from those in the high- $\gamma$ clusters. This appears to be true (Freire et al. 2005; Hessels et al. 2007; Lynch et al. 2011, 2012), but it could be refuted (or further confirmed) by timing more of the pulsars in those clusters - and finding new ones.

\subsection{An intermediate mass black hole in the centre of 47 Tuc?}

Recently, the possibility of an intermediate mass $\left(2300 M_{\odot}\right)$ black hole (IMBH) at the centre of 47 Tuc has been raised considering only the $\dot{P}_{\text {obs }}$ of the pulsars (see Kiziltan et al. 2017 and associated Corrigendum) which give us upper limits on the pulsar accelerations $\left(a_{\ell, \max }\right)$ via Eq, 3. In this work, we consider not only the $a_{\ell \text {, max }}$, but also measurements of jerk along the line of sight $\left(\dot{a}_{\ell}\right)$, and actual measurements of the line-of-sight accelerations in the field of the cluster $\left(a_{\ell, \mathrm{GC}}\right)$ for 10 binary pulsars, as discussed in previous sections. This is important because these accelerations are more constraining than the $a_{\ell \text {, max }}$ taken into account in Kizıltan et al. (2017).

The simple analytical cluster model described in Section 4 with $d=4.69 \mathrm{kpc}$ can account for all the $a_{\ell}$, GC despite the fact that these are more constraining than the $a_{\ell, \max }$. In the cases where these are missing, the model can account for all the $a_{\ell, \max }$ (from the $\dot{P}_{\text {obs }}$ ) as well. Furthermore, the model also accounts for the jerks observed for all the pulsars that lie (in projection) in the core. Thus, considering all the available observations, we come to the conclusion that using our cluster model we find no clear evidence for the existence of an IMBH at the centre of the cluster: its gravity is not necessary to explain the observations.

As shown in section 4 , the cluster distance is crucial for the interpretation of the accelerations. Once we know the standard deviation of the HST proper motions near the centre of the cluster, then the predicted accelerations are proportional to the assumed distance. With the smaller distance assumed by Kizıltan et al. (2017), which we believe to be an under-estimate (see section 1.1), our cluster model is unable to account for the observed accelerations of 47 Tuc S, $\mathrm{E}$ and $\mathrm{U}$.

We must, however, emphasize that this discussion is based on our analytical model, which is not necessarily an accurate description of the actual cluster potential, particularly if a massive black hole is present. A probabilistic estimate of the mass of this hypothetical IMBH will be presented by Abbate et al. (in preparation). 


\section{SUMMARY AND CONCLUSIONS}

In this paper, we have reported on the discovery of two millisecond pulsars in the GC 47 Tucanae (47 Tuc X and Y) and presented 20 timing solutions for just as many pulsars in the cluster. Seventeen of these are updates of previous timing solutions, with more than 10 years of high-resolution data added, and, in the case of 47 Tuc ab, an extended set of ToAs compared to that of Pan et al. (2016). The remaining three solutions (for pulsars 47 Tuc R, Y and Z) are presented here for the first time. With the solutions presented in Ridolfi et al. (2016) (for 47 Tuc W and X) and in Freire \& Ridolfi(2017) (for 47 Tuc aa), we now have a total of 23 timing solutions.

The large timing baseline with uniform coverage from the high-resolution analogue filterbank at Parkes has produced a great improvement in the measurement of several key parameters for these pulsars, in particular those that are relevant for a study of the dynamics of the cluster. The $\dot{P}_{\text {obs }}$ have been used in previous studies of the mass model of 47 Tuc (Freire et al. 2003). In this paper we refine them and present new measurements for pulsars 47 Tuc R, Y and Z. Apart from this, we present several new additional pulsar parameters that are important for a study of the dynamics of the cluster: the proper motions, the real line-of-sight accelerations as determined from the orbital period derivatives and the jerks. All of these will contribute to more detailed analyses such as that done by Prager et al. (2016) for the GC Terzan 5. These should, in particular, be able to a) link the acceleration model with the measured proper motions, something we have not done in this paper, b) provide better probabilistic distance estimates from the acceleration data and c) investigate the probability distribution for the mass of a hypothetical IMBH at the centre of the cluster.

Nevertheless, we can already derive some preliminary conclusions, based on the stellar proper motion dispersion near the centre of the cluster and the analytical cluster acceleration model presented in Section 4: The measurements of acceleration based on $\dot{P}_{\mathrm{b} \text {,obs }}$ can be accounted for by this model with a cluster distance of $4.69 \mathrm{kpc}$. This coincides with most photometric and spectroscopic distances published to date, see e.g. Woodley et al. (2012) and references therein; this suggests that the published $\sigma_{0}$ and the kinematic distances are too small. The likely reasons for this have already been discussed in Bogdanov et al. (2016) and are summarized in the Introduction. If we instead use the smaller distances suggested by kinematic studies then the cluster model is unable to predict the line-of-sight accelerations of three binary pulsars, 47 Tuc E, S, and U.

Regarding the jerks, we find that the cluster potential can also account for most observed jerks, particularly those in the core. Only 47 Tuc H, U and J (which lie well outside the core) have jerks that cannot be explained by any cluster model, these pulsars are clearly being influenced by nearby objects.

The fact that our analytical model with $d=4.69 \mathrm{kpc}$ can account for all observed pulsar accelerations and upper limits and all pulsar jerks in the core means that, with this model, we find no evidence for any excess accelerations near the centre of the cluster such as should be caused by the presence of an intermediate mass black hole.

We have also described the behaviour of the four black widow systems with known timing solutions, 47 Tuc I, J, O and R. Although two of the systems (47 Tuc J and O) exhibit strong variability in their orbital periods, as observed in the long-term timing of other such systems (Shaifullah et al. 2016), two others (47 Tuc I and R) appear to be stable, with orbital period derivatives that are very similar to those expected from their acceleration in the field of the cluster. This hints at a bi-modal behaviour of the black widow systems. If confirmed by the study of other systems, this is important for a variety of applications: some black widows might be suitable for use in pulsar timing arrays.

We can now derive an improved value for the total mass of 47 Tuc H. However, it is not yet possible to measure the masses of the individual components of that system. Furthermore, we have detected the rate of advance of periastron for three more systems (47 Tuc E, S and U), but these are not yet precise enough for astrophysically interesting constraints on the total mass.

\section{ACKNOWLEDGEMENTS}

PCCF and AR gratefully acknowledge financial support by the European Research Council for the European Research Council Starting grant BEACON under contract no. 279702, and continuing support from the Max Planck Society. AR is member of the International Max Planck research school for Astronomy and Astrophysics at the Universities of Bonn and Cologne and acknowledges partial support through the Bonn-Cologne Graduate School of Physics and Astronomy. PT gratefully acknowledges financial support from the European Research Council for the SynergyGrant BlackHoleCam (ERC-2013-SyG, Grant Agreement no. 610058). COH acknowledges funding from an Natural Sciences and Engineering Research Council of Canada Discovery Grant, a Discovery Accelerator Supplement, and an Alexander von Humboldt Fellowship. The Parkes radio telescope is part of the Australia Telescope, which is funded by the Commonwealth of Australia for operation as a National Facility managed by the Commonwealth Scientific and Industrial Research Organisation. DRL was supported by National Science Foundation RII Track I award number 1458952. We especially thank Ralph Eatough for observations, significant assistance with the data handling and for stimulating discussions on pulsar searches, Norbert Wex for important comments and help with the whole project and the referee Scott Ransom for carefully reviewing this work and providing suggestions that improved and shortened it. This research has made use of NASA's Astrophysics Data System.

\section{REFERENCES}

Bahramian A., Heinke C. O., Sivakoff G. R., Gladstone J. C., 2013, ApJ, 766, 136

Bogdanov S., Grindlay J. E., Heinke C. O., Camilo F., Freire P. C. C., Becker W., 2006, ApJ, 646, 1104

Bogdanov S., Heinke C. O., Özel F., Güver T., 2016, ApJ, 831, 184

Brogaard K., VandenBerg D. A., Bedin L. R., Milone A. P., Thygesen A., Grundahl F., 2017, preprint, (arXiv:1702.03421) 
Cadelano M., Pallanca C., Ferraro F. R., Salaris M., Dalessandro E., Lanzoni B., Freire P. C. C., 2015, ApJ, 812, 63

Camilo F., Lorimer D. R., Freire P., Lyne A. G., Manchester R. N., 2000, ApJ, 535, 975

Corongiu A., Possenti A., Lyne A. G., Manchester R. N., Camilo F., D'Amico N., Sarkissian J. M., 2006, ApJ, 653, 1417

D'Amico N., Possenti A., Fici L., Manchester R. N., Lyne A. G., Camilo F., Sarkissian J., 2002, ApJ, 570, L89

Damour T., Deruelle N., 1985, Ann. Inst. Henri Poincaré Phys. Théor., Vol. 43, No. 1, p. 107 - 132, 43, 107

Damour T., Deruelle N., 1986, Ann. Inst. Henri Poincaré Phys. Théor., Vol. 44, No. 3, p. 263 - 292, 44, 263

Damour T., Taylor J. H., 1991, ApJ, 366, 501

Edmonds P. D., Gilliland R. L., Heinke C. O., Grindlay J. E., Camilo F., 2001, ApJ, 557, L57

Edmonds P. D., Gilliland R. L., Camilo F., Heinke C. O., Grindlay J. E., 2002, ApJ, 579, 741

Folkner W. M., Williams J. G., Boggs D. H., 2009, Interplanetary Network Progress Report, 178, 1

Freire P. C. C., 2005, in Rasio F. A., Stairs I. H., eds, Astronomical Society of the Pacific Conference Series Vol. 328, Binary Radio Pulsars. p. 405 (arXiv:astro-ph/0404105)

Freire P. C. C., 2013, in van Leeuwen J., ed., IAU Symposium Vol. 291, Neutron Stars and Pulsars: Challenges and Opportunities after 80 years. pp 243-250 (arXiv:1210.3984), doi:10.1017/S1743921312023770

Freire P. C., Kramer M., Lyne A. G., 2001a, MNRAS, 322, 885

Freire P. C., Camilo F., Lorimer D. R., Lyne A. G., Manchester R. N., D'Amico N., 2001b, MNRAS, 326, 901

Freire P. C., Kramer M., Lyne A. G., Camilo F., Manchester R. N., D'Amico N., 2001c, ApJ, 557, L105

Freire P. C., Camilo F., Kramer M., Lorimer D. R., Lyne A. G., Manchester R. N., D'Amico N., 2003, MNRAS, 340, 1359

Freire P. C. C., Hessels J. W. T., Nice D. J., Ransom S. M., Lorimer D. R., Stairs I. H., 2005, ApJ, 621, 959

Freire P. C. C., et al., 2011, Science, 334, 1107

Grindlay J. E., Heinke C., Edmonds P. D., Murray S. S., 2001, Science, 292, 2290G

Grindlay J. E., Camilo F., Heinke C. O., Edmonds P. D., Cohn H., Lugger P., 2002, ApJ, 581, 470

Hansen B. M. S., et al., 2013, Nature, 500, 51

Harris W. E., 1996, AJ, 112, 1487

Heinke C. O., Grindlay J. E., Edmonds P. D., Cohn H. N., Lugger P. M., Camilo F., Bogdanov S., Freire P. C., 2005, ApJ, 625, 796

Hessels J. W. T., Ransom S. M., Stairs I. H., Freire P. C. C., Kaspi V. M., Camilo F., 2006, Science, 311, 1901

Hessels J. W. T., Ransom S. M., Stairs I. H., Kaspi V. M., Freire P. C. C., 2007, ApJ, 670, 363

Hessels J., et al., 2015, Advancing Astrophysics with the Square Kilometre Array (AASKA14), p. 47

Hotan A. W., van Straten W., Manchester R. N., 2004, Publ. Astron. Soc. Australia, 21, 302

Jacoby B. A., Cameron P. B., Jenet F. A., Anderson S. B., Murty R. N., Kulkarni S. R., 2006, ApJ, 644, L113

Johnson T. J., et al., 2013, ApJ, 778, 106

Joshi K. J., Rasio F. A., 1997, ApJ, 479, 948

King I., 1962, AJ, 67, 471

Kızıltan B., Baumgardt H., Loeb A., 2017, Nature, 542, 203

Knight H. S., 2007, PhD thesis, Swinburne University of Technology

Lange C., Camilo F., Wex N., Kramer M., Backer D. C., Lyne A. G., Doroshenko O., 2001, MNRAS, 326, 274

Lorimer D. R., Camilo F., Freire P., Kramer M., Lyne A. G., Manchester R. N., D'Amico N., 2003, in Bailes M., Nice D. J., Thorsett S. E., eds, Astronomical Society of the Pacific Conference Series Vol. 302, Radio Pulsars. p. 363 (arXiv:astro$\mathrm{ph} / 0210460)$
Lynch R. S., Ransom S. M., Freire P. C. C., Stairs I. H., 2011, ApJ, 734, 89

Lynch R. S., Freire P. C. C., Ransom S. M., Jacoby B. A., 2012, ApJ, 745, 109

Lyne A. G., Brinklow A., Middleditch J., Kulkarni S. R., Backer D. C., Clifton T. R., 1987, Nature, 328, 399

Manchester R. N., Lyne A. G., Johnston S., D'Amico N., Lim J., Kniffen D. A., 1990, Nature, 345, 598

Manchester R. N., Lyne A. G., Robinson C., Bailes M., D’Amico N., 1991, Nature, 352, 219

Manchester R. N., Hobbs G. B., Teoh A., Hobbs M., 2005, AJ, 129, 1993

McLaughlin D. E., Anderson J., Meylan G., Gebhardt K., Pryor C., Minniti D., Phinney S., 2006, ApJS, 166, 249

Özel F., Freire P., 2016, ARA\&A, 54, 401

Pan Z., Hobbs G., Li D., Ridolfi A., Wang P., Freire P., 2016, MNRAS, 459, L26

Phinney E. S., 1992, Philosophical Transactions of the Royal Society of London Series A, 341, 39

Phinney E. S., 1993, in Djorgovski S. G., Meylan G., eds, Structure and Dynamics of Globular Clusters. Astronomical Society of the Pacific Conference Series, pp 141-169

Prager B., Ransom S., Freire P., Hessels J., Stairs I., Arras P., Cadelano M., 2016, preprint, (arXiv: 1612.04395)

Ransom S. M., Hessels J. W. T., Stairs I. H., Freire P. C. C., Camilo F., Kaspi V. M., Kaplan D. L., 2005, Science, 307, 892

Rasio F. A., Pfahl E. D., Rappaport S., 2000, ApJ, 532, L47

Reid M. J., et al., 2014, ApJ, 783, 130

Ridolfi A., et al., 2016, MNRAS, 462, 2918

Rivera-Sandoval L. E., et al., 2015, MNRAS, 453, 2707

Roberts M. S. E., 2013, in van Leeuwen J., ed., IAU Symposium Vol. 291, Neutron Stars and Pulsars: Challenges and Opportunities after 80 years. pp 127-132 (arXiv: 1210.6903), doi:10.1017/S174392131202337X

Robertson H. P., 1938, Ann. Math., 38, 101

Robinson C., Lyne A. G., Manchester R. N., Bailes M., D'Amico N., Johnston S., 1995, MNRAS, 274, 547

Shaifullah G., et al., 2016, MNRAS, 462, 1029

Shklovskii I. S., 1970, Soviet Ast., 13, 562

Smits R., Lorimer D. R., Kramer M., Manchester R., Stappers B., Jin C. J., Nan R. D., Li D., 2009, A\&A, 505, 919

Spitzer L., 1987, Dynamical evolution of globular clusters

Taylor J. H., 1992, Philosophical Transactions of the Royal Society of London Series A, 341, 117

Taylor J. H., Weisberg J. M., 1982, ApJ, 253, 908

Turk P. J., Lorimer D. R., 2013, MNRAS, 436, 3720

Verbunt F., Freire P. C. C., 2014, A\&A, 561, A11

Verbunt F., Hut P., 1987, in Helfand D. J., Huang J.-H., eds, IAU Symposium Vol. 125, The Origin and Evolution of Neutron Stars. p. 187

Watkins L. L., van der Marel R. P., 2016, preprint, (arXiv:1611.03170)

Watkins L. L., van der Marel R. P., Bellini A., Anderson J., 2015a, ApJ, 803, 29

Watkins L. L., van der Marel R. P., Bellini A., Anderson J., 2015b, ApJ, 812, 149

Woodley K. A., et al., 2012, AJ, 143, 50

van Straten W., Bailes M., 2011, Publ. Astron. Soc. Australia, 28,1

van Straten W., Demorest P., Oslowski S., 2012, Astronomical Research and Technology, 9, 237

This paper has been typeset from a $\mathrm{T}_{\mathrm{EX}} / \mathrm{IAT}_{\mathrm{EX}}$ file prepared by the author. 